\title{
Effects of Inclusion of Adjoint Sea Ice Rheology on Backward Sensitivity Evolution Examined Using an Adjoint Ocean-Sea Ice Model
}

\author{
Takahiro Toyoda, Nariaki Hirose, L. Shogo Urakawa, Hiroyuki Tsujino, \\ Hideyuki NaKano, Norihisa Usui, Yosuke FuJi, Kei SAKamoto, \\ AND GORO YAMANAKA \\ Meteorological Research Institute, Japan Meteorological Agency, Tsukuba, Japan
}

(Manuscript received 4 June 2018, in final form 28 February 2019)

\begin{abstract}
As part of the ongoing development of an ocean data assimilation system for operational ocean monitoring and seasonal prediction, an adjoint sea ice model was developed that incorporates sea ice rheology, which was omitted from previously developed adjoint models to avoid model instability. The newly developed adjoint model was merged with the existing system to construct a global ocean-sea ice adjoint model. A series of sensitivity experiments, in which idealized initial values were given for the adjoint sea ice area fraction and thickness, were conducted, with particular attention to the differences between the cases with free-drift approximation in the adjoint sea ice model as in previous studies and with full sea ice dynamics including rheology. The internal stress effects represented in the adjoint rheology induced remarkable differences in the evolution of the initialized and generated adjoint variables, such as for the sea ice velocity by $O\left(10^{2}\right)$ in magnitude, which highlighted the importance of the adjoint rheology in the central Arctic Ocean. In addition, sensitivities with respect to the nonprognostic variables associated with the sea ice dynamics were obtained only through the adjoint rheology. These results suggested a potential for providing an improved global atmosphere-ocean-sea ice state estimation through a four-dimensional variational approach with the adjoint sea ice model as developed in this study.
\end{abstract}

\section{Introduction}

Sea ice is an important component of the polar ocean climate system that greatly affects air-sea heat exchange. For example, the high albedos of sea ice and of snow on top of the ice, compared with the open ocean, reduce absorption of shortwave radiation by the oceansea ice system. Latent heat release to the atmosphere is also greatly reduced when the ocean surface is covered by sea ice with a large dry surface area, and the sensible heat flux and upward longwave radiation may also be moderated because the temperature variation of sea ice is not limited by the freezing point (e.g., Serreze and Barry 2005). Air-sea momentum flux and wave breaking at the surface are also affected by the presence of sea ice (e.g., McPhee 2008). Hence, the effect of sea ice on the air-sea interaction fundamentally influences heat and freshwater circulation in the Arctic Ocean climate. Since the short-term atmospheric variability highly depends on sea ice condition, the accurate prediction of sea ice is

Corresponding author: Takahiro Toyoda, ttyoda@mri-jma.go.jp necessary for the weather prediction in the Arctic Ocean (e.g., Pellerin et al. 2004).

Modeling studies are one way to investigate the role played by sea ice in the Earth system. The advantages of modeling approaches are that all model variables are available for analyzing processes, and predictions as well as simulations of present and past states can be conducted; however, dynamical models inevitably suffer from errors. In particular, model performances are relatively poorer at high latitudes than at lower latitudes, partly because the dynamical scale (e.g., the Rossby deformation radius) is smaller at high latitudes. Errors also result from the parameterizations of processes at high latitudes in the atmosphere (e.g., dominant low-level clouds), the ocean (e.g., intense dense water formation), and sea ice. Sea ice models in particular include many empirical constants ("tuning parameters") that are not determined theoretically

Publisher's Note: This article was revised on 28 May 2019 to correct an editing error in the second paragraph of section 2. 
and which sometimes have nonlinear or compensating effects (e.g., Steele et al. 1997). In coupled ocean-sea ice models, large uncertainties of surface forcing data (mainly obtained from atmospheric reanalyses) (e.g., Lindsay et al. 2014) can be a serious source of error. To reduce such model errors, the resolution and parameterizations of both individual and coupled atmosphere-ocean-sea ice processes need to be improved.

At the Meteorological Research Institute (MRI), an ocean general circulation model (OGCM) and ocean data assimilation system have been developed to enhance the accuracy of operational ocean monitoring and seasonal predictions by the Japan Meteorological Agency (e.g., Usui et al. 2006). To improve the representation of Arctic Ocean variability, sophisticated parameterizations for dynamical and thermodynamical sea ice processes have been embedded in the OGCM (Tsujino et al. 2011), and the global version of the data assimilation system has been updated to include the Arctic Ocean (Toyoda et al. 2013).

Recently, Toyoda et al. (2016) developed a scheme for assimilating sea ice concentration (SIC) data into the MRI system based on the three-dimensional variational data assimilation (3DVAR) method. The scheme uses observational data to correct both the SIC field and surface boundary conditions (air temperature and specific humidity). The inclusion of the latter was motivated by a preliminary experiment in which, although the model SIC was greatly changed by assimilation of SIC itself (without atmospheric correction), the ocean-sea ice surface temperature (area averaged ice-top and openwater temperatures within a grid cell) changed little because it was strongly constrained by surface air temperature. Instead, the assimilation increments were compensated by changes in other variables without data constraint (e.g., sea ice thickness), which resulted in a great thermodynamical imbalance without the increments. In the developed scheme, the correction of the surface air temperature was estimated from the area fraction change of open water and sea ice (with respective top temperatures) by the assimilation. The constant offsets (corrections) imposed on the surface boundary conditions worked to retain the natural thermodynamical balance with the analyzed sea ice field. The simple, but effective, approach adopted in that study resulted in the enhanced reproducibility of SIC and related fields, such as sea ice thickness and oceanic freshwater content.

An adjoint model approach is one way to achieve more comprehensive correction of model variables, including surface boundary conditions and uncertain model parameters, to further improve model reproducibilities. Thus, we have a high motivation to improve the state estimation through the four-dimensional variational (4DVAR) method. Note that the above result also suggested the great potential of coupled atmosphere-ocean-sea ice adjoint model approaches (e.g., Fujii et al. 2018).

Here, we developed an adjoint model for the sea ice part (as well as the air-ocean surface flux part) of MRI's OGCM that was eventually merged with the main adjoint model developed previously (Usui et al. 2015). Our adjoint model includes both the thermodynamics and dynamics of sea ice. Behavior of an adjoint of full sea ice dynamics including rheology has not been documented before mainly because previous adjoint models greatly simplified sea ice dynamics (free drift) to avoid numerical instabilities (Fenty and Heimbach 2013). It would be necessary to understand the behavior of an adjoint of a sea ice system with full sea ice dynamics before we apply it to a more complex ocean-sea ice 4DVAR data assimilation system. Thus, we examined the effects of including the full sea ice dynamics in our system in this study.

We used our adjoint sea ice model to investigate the backward time evolution of sea ice sensitivities in the following manner. First, initial (nonzero) values of adjoint variables for the sea ice area fraction and thickness were given at characteristic points for the marginal ice zone and the central Arctic Ocean, mimicking the impact of having observations at those characteristic points. Then, initial values with a basin-scale distribution were employed to further investigate the effects of the improved adjoint sea ice dynamics. Although the sensitivity experiments in this study are more idealized than those of previous studies (e.g., Kauker et al. 2009; Fenty and Heimbach 2013; Kauker 2015; Koldunov et al. 2017), the results of our investigation on time evolutions of both initialized and not initialized adjoint variables, especially via the adjoint sea ice dynamics, are expected to provide valuable information to the sea ice data assimilation community for improving assimilation systems.

In addition, the adjoint model includes adjoint variables for uncertain model parameters as well as surface boundary conditions. Considering the possible error sources in ocean-sea ice models, the sensitivities of sea ice variables to these uncertain parameters (and the use of these sensitivities to adjust these parameters in future data assimilation experiments) are as important as their sensitivities to initial and boundary conditions addressed in previous studies. Parameter tuning by trial and error has been adopted even for numerical models used in data assimilation experiments, although a relatively simple Green's function approach was also adopted to adjust ocean-sea ice model parameters (Nguyen et al. 2011). 
The structure of this article is as follows: both the forward and adjoint models are described in section 2, the results of sensitivity experiments are presented and discussed in section 3, a further discussion is made in section 4 , and a summary is provided in section 5 .

\section{Forward and adjoint models}

\section{a. $O G C M$}

We used the MRI Community Ocean Model (MRI.COM) version 4.0 (Tsujino et al. 2017) as the OGCM. A sea ice model embedded in the OGCM describes both thermodynamics and dynamics. The thermodynamical part is based on Mellor and Kantha (1989) and considers the heat capacity of sea ice (one-layer ice). The sea ice dynamics is based on the elastic-viscousplastic rheology of Hunke and Dukowicz (1997). The empirical constants for sea ice strength (pressure) (Hibler 1979) are set to $P^{*}=2.75 \times 10^{4} \mathrm{Nm}^{-2}$ and $C=20$ (Hibler and Walsh 1982). In addition to the rheology, a parameterization for the evolution of the sea ice thickness distribution within a grid cell due to ridging (and other mechanical processes) (Thorndike et al. 1975) is adopted, as in Community Ice Code (CICE), the Los Alamos sea ice model (Hunke and Lipscomb 2010). The constant representing the high end of the area fraction subject to this process is set to $G^{*}=0.15$, whereas the fraction of shear dissipation energy that contributes to ridge building (Flato and Hibler 1995) is set to $C_{S}=0.5$. Details of the sea ice dynamics parameterizations are described below.

Other factors were also taken from CICE. Five sea ice thickness categories are allowed within a grid cell (Lipscomb 2001): 0.0-0.6, 0.6-1.4, 1.4-2.4, 2.4-3.6, and $3.6-30.0 \mathrm{~m}$. Albedo values for near-infrared $(>700 \mathrm{~nm})$ and visible wavelengths $(<700 \mathrm{~nm})$ are defined for both sea ice ( 0.36 and 0.78 , respectively) and snow ( 0.70 and 0.98 , respectively), with rates of decrease (air temperature $>-1{ }^{\circ} \mathrm{C}$ ) of $-0.075\left({ }^{\circ} \mathrm{C}\right)^{-1}$ for sea ice (for both near-infrared and visible wavelengths) and of $-0.15\left({ }^{\circ} \mathrm{C}\right)^{-1}\left[-0.10\left({ }^{\circ} \mathrm{C}\right)^{-1}\right]$ for snow for near-infrared (visible) wavelengths. Salinity in sea ice, reflecting the presence of brine, is set to 4.0. The air-ice drag coefficient is set to $C_{d}=1.63 \times 10^{-3}$. See Tsujino et al. (2017) for details of these parameterizations and parameter values.

We used the GONDOLA_100 experimental framework (Urakawa et al. 2017), which covers the whole global ocean, to conduct our experiments. In this framework, the horizontal resolution south of $64^{\circ} \mathrm{N}$ is $1^{\circ}$ of longitude and $0.3^{\circ}-0.5^{\circ}$ of latitude (finer around the equator). North of $64^{\circ} \mathrm{N}$, a bipolar grid (Murray 1996) is adopted with polar singularities at $64^{\circ} \mathrm{N}, 80^{\circ} \mathrm{E}$ and $64^{\circ} \mathrm{N}, 100^{\circ} \mathrm{W}$ on land. A total of 60 vertical levels and a bottom boundary layer are defined. The model is driven by downward shortwave and longwave radiation, precipitation, 10-m air temperature, specific humidity, and $10-\mathrm{m}$ wind speed and associated direction fields, all of which are calculated from the 3-hourly JRA55-do dataset. This dataset, which is based on the JRA-55 atmospheric reanalysis (Kobayashi et al. 2015) and includes corrections for global heat and freshwater budget balances, was constructed specifically to provide forcing data for driving ocean models (hence the abbreviation JRA55-"do"; Tsujino et al. 2018). Latent and sensible heat fluxes and surface stresses are calculated from the bulk formulas of Large and Yeager (2009).

The initial conditions for the below sensitivity experiments were taken from the data assimilative simulation from 1958 by using JRA55-do. In this simulation, a 3DVAR method with a 10-day assimilation window (see Toyoda et al. 2016) was used to assimilate in situ temperature and salinity profiles (Boyer et al. 2013; Good et al. 2013) and satellite-based sea surface temperature data (Kurihara et al. 2006).

\section{b. Adjoint model}

Usui et al. (2015) have described the main adjoint model for MRI.COM. This system has already been used for 4DVAR state estimation experiments (e.g., Usui et al. 2017). The main adjoint model was recently revised in conjunction with the updating of MRI.COM from version 2.4 to 4.0 (Tsujino et al. 2017).

As described above, the sea ice model consists of the thermodynamical and dynamical parts. 1) Intermediate conditions due to fluxes (of heat, freshwater and salt) on the top and bottom of ice/snow are first calculated in the thermodynamical part. 2) In the dynamical part, sea ice velocities are determined by using wind and ocean surface stresses and sea ice internal stresses through the rheology. Note that effects of the internal stresses are eliminated if free drift is assumed. 3) Horizontal transports due to advection and diffusion are calculated by using the sea ice velocities to modify the sea ice and snow conditions resulting from the above thermodynamical part. 4) In addition, mechanical ridging effect, which is calculated from the internal stresses, changes the sea ice distribution within a grid. 5) Moreover, the part of snow that is below freeboard becomes sea ice. 6) Finally, sea ice is redistributed into the thickness categories within a grid. Dynamical part is composed of parts 2,3, and 4, while thermodynamical part (including redistribution) is composed of part 1,5 , and 6 . The adjoint of the sea ice model thus consists of the adjoint of these components. 
Adjoint sea ice models that have been developed for systems of other research institutes (e.g., Kauker et al. 2009) have used an automatic differentiation tool called Transformation of Algorithms in FORTRAN (Giering and Kaminski 1998). The adjoint sea ice model in this study was written manually but the methodology is basically the same as that used previously.

Here, we describe an adjoint sea ice model that includes the rheology. ${ }^{1}$ In the elastic-viscous-plastic rheology model of Hunke and Dukowicz (1997), the constitutive law is

$$
\frac{1}{E} \frac{\partial \sigma_{i j}}{\partial t}+\frac{1}{2 \eta} \sigma_{i j}+\frac{\eta-\zeta}{4 \eta \zeta} \sigma_{k k} \delta_{i j}+\frac{P}{4 \zeta} \delta_{i j}=\dot{\epsilon}_{i j},
$$

where $E$ is the Young's modulus, $\sigma_{i j}$ is the stress tensor, $\delta_{i j}$ is the Kronecker delta $\left(\delta_{i j}=1\right.$ if $i=j$, otherwise 0$)$, and $\dot{\epsilon}_{i j}$ is the strain rate tensor; $\zeta$ and $\eta$ represent the bulk and shear viscosities, defined as

$$
\begin{gathered}
\zeta=\frac{P}{2 \Delta}, \\
\eta=\frac{P}{2 e^{2} \Delta},
\end{gathered}
$$

where

$$
\Delta=\left[\left(\dot{\epsilon}_{11}^{2}+\dot{\epsilon}_{22}^{2}\right)\left(1+e^{-2}\right)+2\left(1-e^{-2}\right) \dot{\epsilon}_{11} \dot{\epsilon}_{22}+4 e^{-2} \dot{\epsilon}_{12}^{2}\right]^{1 / 2} .
$$

Here, $e$ represents the ratio of normal stress to shear stress (set to 2; Hibler 1979), and $P$ is the ice strength parameter, defined as

$$
P=P^{*} H \exp [-C(1-A)],
$$

where $P^{*}$ and $C$ are parameters defined empirically and $A$ and $H$ represent the sea ice area fraction and thickness, respectively. In this study, $A$ is the sum of the sea ice area fractions of the five thickness categories in a grid cell, and $H$ is the average of the categorized sea ice thicknesses in a grid cell (i.e., $A=\sum_{n=1}^{5} a_{n}$ and $H=\sum_{n=1}^{5} a_{n} h_{n}$, where $a_{n}$ and $h_{n}$ are area fraction and thickness in category $n$, respectively). Sea ice velocity field is eventually calculated by using the stress tensor

\footnotetext{
${ }^{1}$ In the review process, an anonymous reviewer indicated that the adjoint of a viscous-plastic rheology model was used by Kauker et al. (2009), although it was not explicitly described in the literature (Kauker et al. 2009; Kauker 2015). Thus, it is considered that the stable adjoint of full sea ice dynamics was first generated for their system.
}

determined through (1). Note that the strain tensor is calculated from sea ice velocity.

The fractional area subject to ridging is (Thorndike et al. 1975)

$$
a(h)=b(h) g(h),
$$

where $h$ is thickness, $g(h)$ is the thickness distribution function, and $b(h)$ is the weighting function, determined as follows:

$$
\begin{aligned}
& b(h)=\frac{2}{G^{*}}\left[1-\frac{G(h)}{G^{*}}\right] \text { for } 0 \leq G(h) \leq G^{*} \\
& b(h)=0 \text { for } G(h)>G^{*}
\end{aligned}
$$

where $G(h)$ is the cumulative thickness distribution $\left[G(h)=\int_{0}^{h} g(h) d h\right]$ and $G^{*}$ is a parameter defined empirically. $G(h)$ includes the open water fraction, so ridging occurs only when the total sea ice area fraction is greater than $1-G^{*}(0.85$ in this study $)$. The net area loss rate for pack ice (due to closing of areas of open water and ridging), $R_{\text {net }}$, is assumed to be a function of the strain rate:

$$
R_{\text {net }}=\frac{C_{S}}{2}\left(\Delta-\left|\dot{\epsilon}_{11}+\dot{\epsilon}_{22}\right|\right)-\min \left(\dot{\epsilon}_{11}+\dot{\epsilon}_{22}, 0\right),
$$

where the first and second terms on the rhs represent the contributions from shear and convergence, respectively. Strain rates are computed in the rheology scheme; $R_{\text {net }}$ is partitioned into the thickness categories according to the fractional contribution of each category obtained by integrating $a(h)$ over the thickness range of the category. The thickness of ridged ice is calculated from its volume by assuming that energy is conserved. The ridged ice is then redistributed into appropriate thickness categories.

The nonlinearity of these equations mainly arises from the fact that, in the rheology, the viscosities ( $\zeta$ and $\eta)$ depend on the strain tensor. Adjoint equations corresponding to (2) and (3) are

$$
\begin{aligned}
& \tilde{P}=\tilde{P}_{0}+\frac{\tilde{\zeta}}{2 \Delta}+\frac{\tilde{\eta}}{2 e^{2} \Delta}, \\
& \tilde{\Delta}=\tilde{\Delta}_{0}-\frac{P \tilde{\zeta}}{2 \Delta^{2}}-\frac{P \tilde{\eta}}{2 e^{2} \Delta^{2}},
\end{aligned}
$$

where $\tilde{P}, \tilde{\Delta}, \tilde{\zeta}$, and $\tilde{\eta}$ are the adjoint variables for $P, \Delta, \zeta$, and $\eta$, respectively, and $\tilde{P}_{0}$ and $\tilde{\Delta}_{0}$ represent contributions from the other parts (e.g., the adjoint ridging part). Note that, since a smaller time step is used in the rheology model than in the main OGCM, these contributions are accumulated during the OGCM's time step. 
We found that stable adjoint model integration was possible by eliminating the second and third terms in the rhs of (11). The elimination of the effect of the adjoint sea ice viscosities [the second and third terms in (11)] is considered to be an analog of the previous studies in which the vertical viscosity and diffusivity parameterizations were skipped in the adjoint models. The influence of the elimination in this study is discussed further in section 4 . Note that $\tilde{\Delta}$ (and $\tilde{\Delta}_{0}$ ) is still used in the adjoint equations for (4) and (9), which represent the connection, in the adjoint model, between the sea ice velocity field determined by the rheology and the ridging process. Thus, the adjoint variables for $R_{\text {net }}$ influences the adjoint sea ice velocity [via the adjoint strain rates through the adjoint equations for both (4) and (9)]. The adjoint equations for (5) are written using $\tilde{P}$ as

$$
\begin{aligned}
\widetilde{P^{*}} & =\tilde{P} H \exp [-C(1-A)], \\
\tilde{C} & =-(1-A) \tilde{P} \times P^{*} H \exp [-C(1-A)], \\
\tilde{H} & =P^{*} \tilde{P} \exp [-C(1-A)], \\
\tilde{A} & =C \tilde{P} \times P^{*} H \exp [-C(1-A)],
\end{aligned}
$$

where $\widetilde{P^{*}}, \tilde{C}, \tilde{H}$, and $\tilde{A}$ are the adjoint variables for $P^{*}, C, H$, and $A$, respectively. Because in preliminary experiments, feedbacks from the dynamical processes sometimes led to instability in $\tilde{A},(15)$ was excluded from the adjoint of (5) in this study. Note that $\tilde{H}$ is related to the adjoint variables for sea ice area fraction and thickness $\widetilde{a_{n}}$ and $\widetilde{h_{n}}$ as $\widetilde{a_{n}}=\tilde{H} h_{n}$ and $\widetilde{h_{n}}=a_{n} \tilde{H}$, respectively. The discretization of (1) is as described by Hunke and Lipscomb (2010), and the tangent linear and corresponding adjoint models are coded based on the discretized model.

We also developed an adjoint model for the surface flux part of our system, in a similar manner as in previous studies (e.g., Awaji et al. 2003), that includes adjoint variables for sea ice and snow albedos and the air-sea ice drag coefficient so that sea ice sensitivities to these parameters could be investigated. In addition, we considered the sensitivities to the parameters for the ridging scheme $\left(G^{*}\right.$ and $\left.C_{S}\right)$.

By merging our adjoint sea ice model with the existing adjoint model, a global ocean-sea ice adjoint model was constructed that includes both the dynamical and thermodynamical parts of the sea ice model. In this model, adjoint variables are defined not only for initial and boundary conditions but also for several tuning parameters. Consistency between the tangent linear and adjoint models was verified by integrating the models

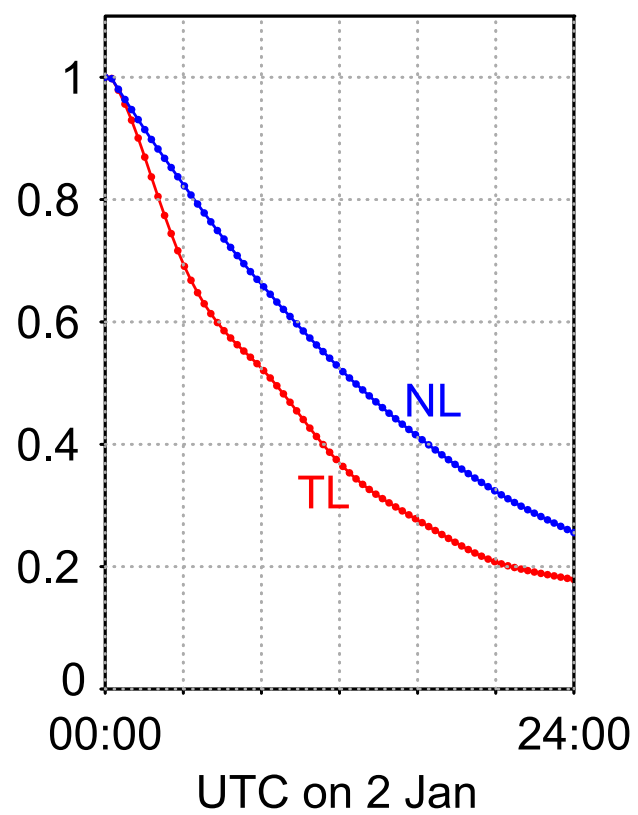

FIG. 1. Time evolutions of finite difference gradients for the sea ice area fraction at a point $\left(80^{\circ} \mathrm{N}, 120^{\circ} \mathrm{W}\right)$ as initialized at $0000 \mathrm{UTC}$ 2 Jan 2000. See section 3 for the point and the day. Blue line indicates the difference between the results with and without perturbation from the nonlinear model, normalized by the magnitude of the initial perturbation $10^{-4}$. Red line indicates the tangent linear evolution of the initialized value 1 .

with perturbations given for all tangent linear/adjoint variables that corresponds to control variables (i.e., initial and boundary conditions and tuning parameters) and many intermediate variables that appear in the integration, as done for the previous system (Usui et al. 2015). The exact consistency was confirmed from the perturbed experiments (using double precision) with 72 time steps (one day). Exponentially growing signals (i.e., numerical instabilities) were not seen during the period. In addition, the consistency between the nonlinear and the tangent linear models was verified by examining finite difference gradients. Thus, evolution of difference between the baseline and perturbed integrations by the nonlinear forward model and evolution of the same perturbation in the integration by the tangent linear forward model were compared (e.g., Kalnay et al. 2012). Note that the above-described consistency guaranteed the same approximation (linearization) on the nonlinear model for the tangent linear and adjoint models. For example, Fig. 1 compares the nonlinear and tangent linear evolutions of finite difference gradients for sea ice area fraction at a point $\left(80^{\circ} \mathrm{N}\right.$, $120^{\circ} \mathrm{W}$ ) during one day ( 2 January 2000 ; see section 3 for the point and the duration). A broad similarity of these time evolutions supports the general correctness of the tangent linear model and hence the adjoint 
TABLE 1. Backward sensitivity experiments. All experiments were conducted with one-day window (2 Jan 2002); $\widetilde{a_{n}}$ and $\widetilde{h_{n}}$ represent the ajoint sea ice area fraction and thickness, respectively, for each category $(n=1-5)$.

\begin{tabular}{|c|c|c|c|}
\hline \multirow[b]{2}{*}{ Name } & \multicolumn{2}{|c|}{ Initial value } & \multirow[b]{2}{*}{ Supplemental setting } \\
\hline & Location & Variable & \\
\hline EXP-Da & Denmark Strait $\left(70^{\circ} \mathrm{N}, 18^{\circ} \mathrm{W}\right)$ & $\widetilde{a_{n}}$ & \\
\hline EXP-Da_sub & & $\widetilde{a_{n}}$ & Without adjoint bottom melting \\
\hline EXP-Dh & & $\widetilde{h_{n}}$ & \\
\hline EXP-DaX & & $\tilde{a_{n}}$ & Reduced adjoint sea ice dynamics \\
\hline EXP-DhX & & $\widetilde{h_{n}}$ & Reduced adjoint sea ice dynamics \\
\hline EXP-Ca & Central Arctic $\left(80^{\circ} \mathrm{N}, 120^{\circ} \mathrm{W}\right)$ & $\tilde{a_{n}}$ & \\
\hline EXP-Ch & & $\widetilde{h_{n}}$ & \\
\hline EXP-CaX & & $\tilde{a_{n}}$ & Reduced adjoint sea ice dynamics \\
\hline EXP-ChX & & $\widetilde{h}_{n}$ & Reduced adjoint sea ice dynamics \\
\hline EXP-Aa & Whole Arctic Ocean & $\widetilde{a_{n}}$ & \\
\hline EXP-Ah & & $\widetilde{h_{n}}$ & \\
\hline EXP-AaX & & $\tilde{a_{n}}$ & Reduced adjoint sea ice dynamics \\
\hline EXP-AhX & & $\widetilde{h_{n}}$ & Reduced adjoint sea ice dynamics \\
\hline
\end{tabular}

model. We investigated the behavior of the adjoint variables in sensitivity experiments, as described in section 3 .

\section{Sensitivity experiments}

We conducted a series of idealized sensitivity experiments (Table 1) with the adjoint model to investigate both the dynamical and thermodynamical evolution of sea ice sensitivities. We used both forward and adjoint models in the sensitivity experiments because variable values provided by the forward model are necessary for the adjoint calculation.

\section{a. Sensitivities in a region where effects of internal stresses are relatively small}

In the first set of experiments, we examined sea ice sensitivities in a marginal ice zone, where the effects of internal stresses (i.e., rheology and ridging parameterizations) are relatively small (i.e., sea ice transport is largely explained by free drift) (e.g., Haas 2003). We selected an experimental period in the freezing season, when thermodynamical processes are less complicated than in the melting season at least in our model (e.g., the albedos of sea ice and snow abruptly reduced with surface air temperature above $-1^{\circ} \mathrm{C}$ ). For expediency, we selected a day in January 2000. Because a free forward run with initial conditions taken from an assimilation experiment results in an initial shock affecting a few time steps, we used the results for the latter half of a 2-day free forward run starting from 0000 UTC 1 January. Hence, we carried out a backward sensitivity experiment from 2400 to 0000 UTC 2 January, with the initial conditions of adjoint variable for sea ice area fraction (hereafter "adjoint sea ice area fraction") set to +0.01 for each category at a point in the Denmark Strait $\left(70^{\circ} \mathrm{N}, 18^{\circ} \mathrm{W}\right.$; point $\mathrm{D}$ hereafter) and 0 for the other adjoint variables and in the other regions. This is mimicking a data-model misfit in sea ice area fraction of 0.01 with a unit observation error for a target function $J=$ $1 /\left(2 \sigma^{2}\right) \sum_{n=1}^{5}\left(a_{n}^{\text {obs }}-a_{n}\right)^{2}$, where $a_{n}^{\text {obs }}$ and $\sigma$ are the observation data and error. Note that an adjoint variable represents the derivative of the target function $J$ with respect to the corresponding forward variable (e.g., $\left.\widetilde{a_{n}}=\partial J / \partial a_{n}\right)$ and, hence, is not limited as the forward variables, such as $[0,1]$ for $a_{n}$. Since an adjoint model is linear by definition, linear combination of the initial conditions as used here and below leads to the result of the sensitivity experiment equal to the linear combination of the results corresponding to the individual initial conditions. Thus, the initial value is arbitrary. For example, results are magnified tenfold exactly with the initial value of 0.1 instead of 0.01 . We considered this experiment (EXP-Da) to represent, by and large, typical marginal sea ice conditions (Fig. 2).

The total sea ice area fraction (Fig. 2a) and the gridaveraged sea ice thickness (Fig. 2c) at point D during the experiment were 0.428 and $0.894 \mathrm{~m}$, respectively. The sea ice was moving southwestward at a speed of $0.140 \mathrm{~m} \mathrm{~s}^{-1}$, and on average sea ice was converging at point $\mathrm{D}$ (Fig. 2c), although intradaily variability with an inertial period of about a half-day was seen. At point $\mathrm{D}$, surface air temperature was near $0^{\circ} \mathrm{C}$ (Fig. 2a) and the net surface heat flux was small. The ocean surface temperature, however, was $-0.593^{\circ} \mathrm{C}$, so the sea ice was melting. Therefore, in all thickness categories, both the sea ice area fraction and the sea ice thickness decreased during the day (Figs. $2 b$ and $2 d$ ). Sea ice pressure (and thus internal stresses) was relatively small, and ridging did not occur (open water area fraction larger than $G^{*}$ ). 
(a) Total SI fraction \& air temperature

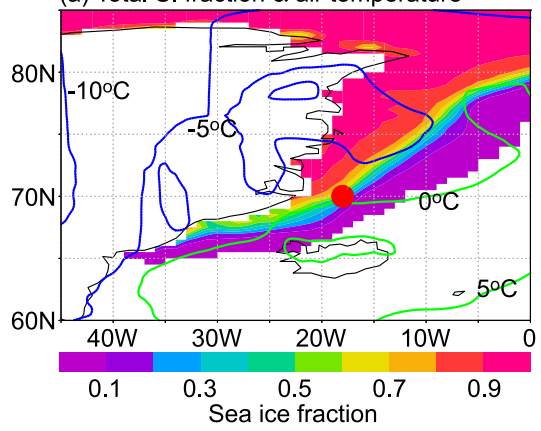

(c) SI thickness \& velocity

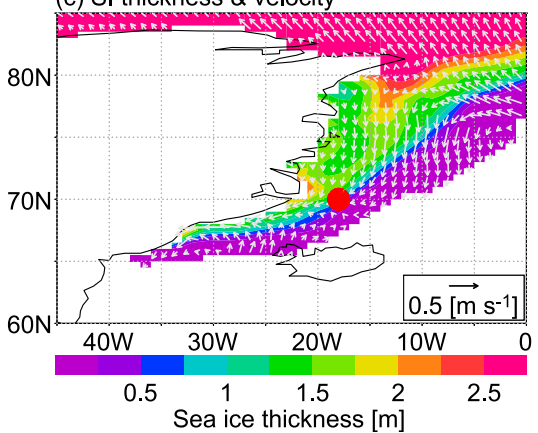

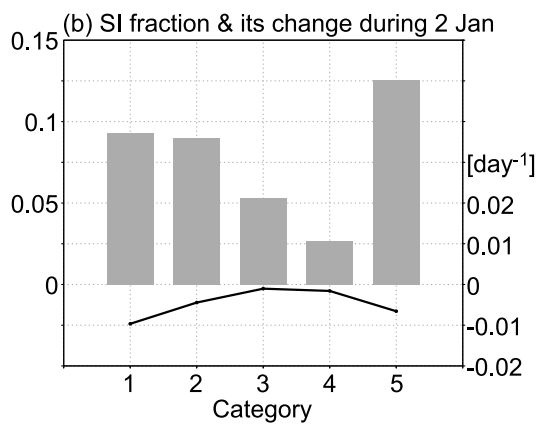

(d) SI thickness \& its change during 2 Jan

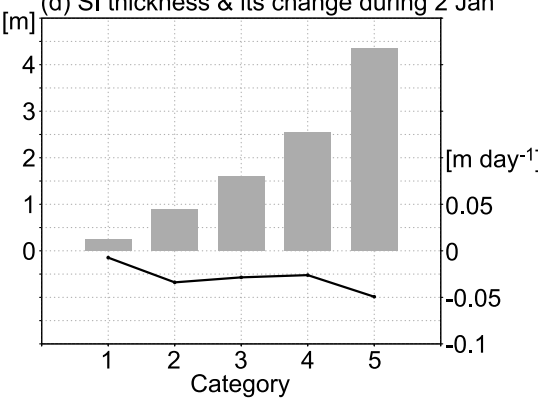

FIG. 2. Forward model variables during the sensitivity experiment (2 Jan 2000). (a) Distribution of the total sea ice (SI) area fraction (color shades) and 10-m air temperature (contours). (b) Sea ice area fraction in each ice thickness category $\left(a_{n}\right.$; bars, left axis) and its change from 0000 to $2400 \mathrm{UTC}$ (line, right axis) at point $\mathrm{D}\left[70^{\circ} \mathrm{N}, 18^{\circ} \mathrm{W}\right.$; solid red circle in (a) and (c)]. (c) Distribution of grid averaged sea ice thickness (color shades) and velocity (arrows). (d) As in (b), but for sea ice thickness $\left(h_{n}\right)$.

During the backward integration, the value of the adjoint sea ice area fraction $\widetilde{a_{n}}$ (where $n$ is the thickness category number) at point $\mathrm{D}$ gradually decreased from its initialized value of +0.01 (Fig. 3a). This decrease can be attributed mainly to advection and diffusion processes, because the value increased at surrounding points. Note that these are only processes that directly connect sea ice values between the grids. In our system, the sea ice area fraction and volume $\left(a_{n}\right.$ and $V_{n} \equiv a_{n} h_{n}$, respectively) in each category are advected (and diffused) and then the resulting values are used to recalculate thickness (as $\left.h_{n}=V_{n} / a_{n}\right)$. The adjoint of this calculation is as follows:

$$
\widetilde{a}_{n}=\widetilde{a}_{n 0}-\frac{V_{n}}{\left(a_{n}\right)^{2}} \times \widetilde{h}_{n},
$$

where $\widetilde{h_{n}}$ is the adjoint variable for sea ice thickness (hereafter "adjoint sea ice thickness") in category $n$ and $\widetilde{a}_{n 0}$ is the contribution from other procedures. (a) Adjoint SI area fraction

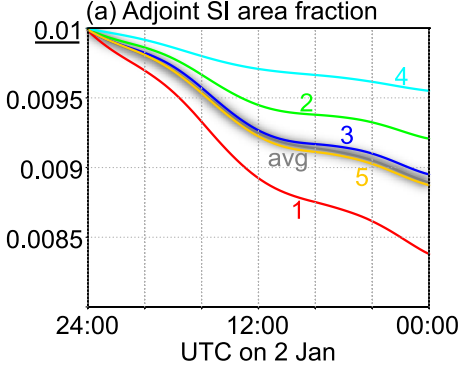

(b) Adjoint SI thickness cat:1

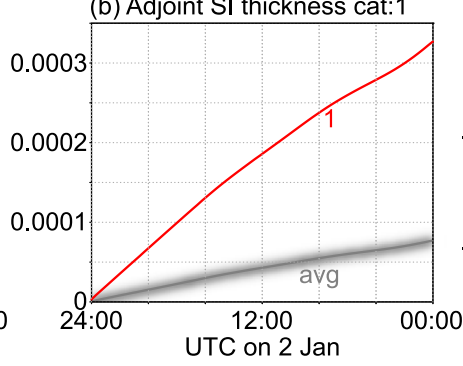

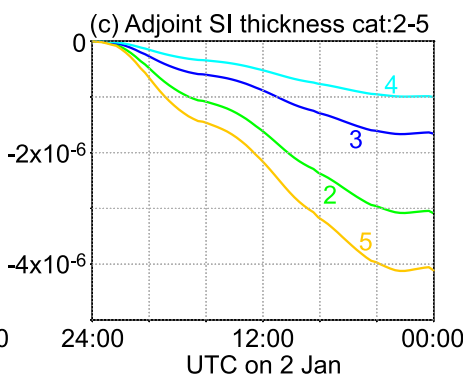

FIG. 3. Backward time evolutions of adjoint sea ice variables at point D in EXP-Da: (a) $\widetilde{a_{n}}$, (b) $\widetilde{h_{1}}$ (red line), and (c) $\tilde{h}_{n}, n=2-5$. Numbers by each line indicate categories. The fraction-weighted grid average values $\tilde{a}$ and $\tilde{h}$ are also plotted in (a) and (b), respectively (gray lines). 
(a) Adjoint SI thickness

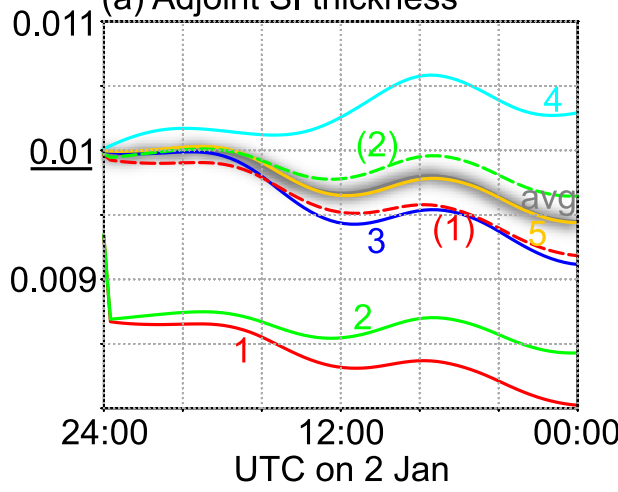

(b) Adjoint SI area fraction

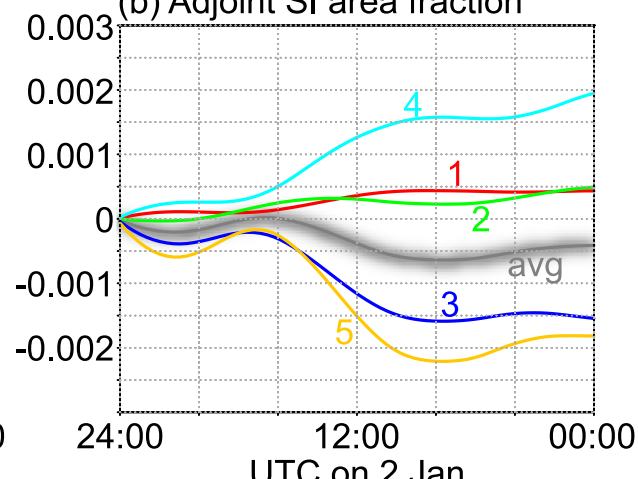

(c) Adjoint $h_{3}$ at 00:00

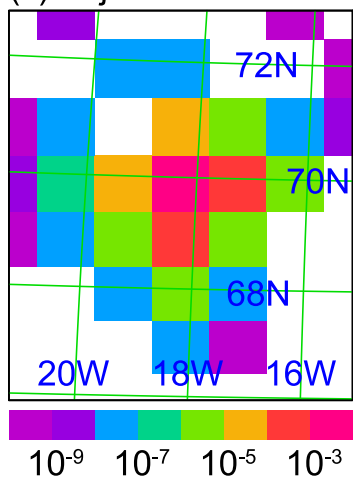

FIG. 4. (a),(b) Backward time evolutions of adjoint sea ice variables at point D in EXP-Dh: (a) $\tilde{h_{n}}$ and $\tilde{h}$ and (b) $\widetilde{a_{n}}$ and $\tilde{a}$. Dashed lines in (a) represent the sum of adjoint sea ice and snow thickness values in categories 1 and 2 (see text). (c) Horizontal distribution of $\widetilde{h_{3}}$ at 0000 UTC around point D in EXP-Dh. Only values greater than $10^{-10}$ are plotted.

The differences in the time evolution of $\widetilde{a}_{n}$ among the categories (Fig. 3a) thus reflect the different contributions from the second term on the rhs of (16). Note that no additional term as (16) appears for $\widetilde{h_{n}}$.

The time evolution among $\widetilde{h_{n}}$, which is forced by the initialized $\widetilde{a_{n}}$ values, shows that $\widetilde{h_{1}}$ increased rapidly (Fig. 3b) compared with smaller decreases in $\vec{h}_{n}$ for $n=2-5$ (Fig. 3c). This difference can be attributed to melting at the bottom of the sea ice, because when we conducted the same experiment without the adjoint of the bottom melting process (EXP-Da_sub), $\widetilde{h_{1}}$ did not show a similar increase. In the forward integration, bottom melting basically reduces the thickness of ice $h_{n}$ in categories 2-5 (Fig. 2d), and the thinned ice is then redistributed to lower (thinner ice) categories. Thus, this redistribution process (within a grid) leads to both decrease of $a_{n}$ and increase of $a_{n-1}$. From the volume conservation, the latter is greater in magnitude. In the backward model integration, the positive $\widetilde{a_{n}}$ and $\widetilde{a_{n-1}}$ values (as initialized) result in increase of $\widetilde{h_{n}}$ and decrease of $\widetilde{h_{n}}$, respectively, with the latter relatively greater in magnitude (due to the similar $\widetilde{a_{n}}$ and $\widetilde{a_{n-1}}$ values), leading to the weak decrease of $\widetilde{h_{n}}$ for $n=2-5$ (Fig. 3c). In the case of ice in category 1 , however, melting primarily reduces the sea ice area fraction rather than the sea ice thickness (Fig. 2b). Because the magnitude of the sea ice area fraction change due to bottom melting in category 1 depends on the thickness (i.e., $h_{1}$ affects the change in $a_{1}$ ) in the forward integration, the sensitivity with respect to thickness in category 1 is forced by the sea ice area fraction increase (i.e., $\widetilde{a_{1}}$ affects $\widetilde{h_{1}}$ ) in the backward integration. The resulting value $\widetilde{h_{n}}$ in turn affects the time evolution of $\widetilde{a_{n}}$ for each category via (16).

In the next experiment (EXP-Dh), an initial value of the adjoint sea ice thickness at point $\mathrm{D}$ of +0.01 was given instead of for the adjoint area fraction. This corresponds to a data-model thickness misfit of $+0.01 \mathrm{~m}$ with a unit observation error $(1 \mathrm{~m})$, for example. In the first step of EXP-Dh, $\widetilde{h_{1}}$ and $\widetilde{h_{2}}$ dropped rapidly (Fig. 4a). In our model, a parameterization was adopted in which the part of snow that is below freeboard absorbs seawater and becomes sea ice. This process affects ice in categories 1 and 2 in the forward integration; thus, a part of the sea ice in these categories became snow in the backward integration. Hence, the sum of the adjoint variables for sea ice and snow thickness (with the latter weighted by their density ratio) did not exhibit a corresponding rapid reduction at the first time step (Fig. 4a, dashed lines). As in EXP-Da, in EXP-Dh, the gross decreasing tendencies of the time series can be explained by the effect of advection and diffusion processes (e.g., Fig. 4c). Note that the effect of melting by oceanic heat is not effective in EXP-Dh. In addition, an oscillatory feature with a period of about a half-day can be seen in Figs. $4 \mathrm{a}$ and $4 \mathrm{~b}$; this feature is considered to reflect the influence of inertial oscillation in the forward model velocity field (e.g., Colony and Thorndike 1980).

The sensitivities also spread to adjoint variables other than those of sea ice. In EXP-Dh, for example, negative values for both adjoint ocean potential temperature and salinity ( $\tilde{T}$ and $\tilde{S}$, respectively) were seen in the surface mixed layer (to approximately 10-15-m depth) (Figs. 5a and $5 \mathrm{~b}$ ). The magnitudes of these values were much greater in EXP-Dh than in EXP-Da (not shown), although the patterns were similar between the two experiments. For example, surface $\tilde{T}(\tilde{S})$ at 0000 UTC in EXP-Dh was larger than the corresponding value in EXP-Da by a factor of $17.5(16.0)$. The negative $\tilde{T}$ and $\tilde{S}$ values resulting from the positive $\widetilde{a_{n}}$ are intuitive, since colder ocean temperature promotes freezing and lower surface salinity increases stratification such that the available 

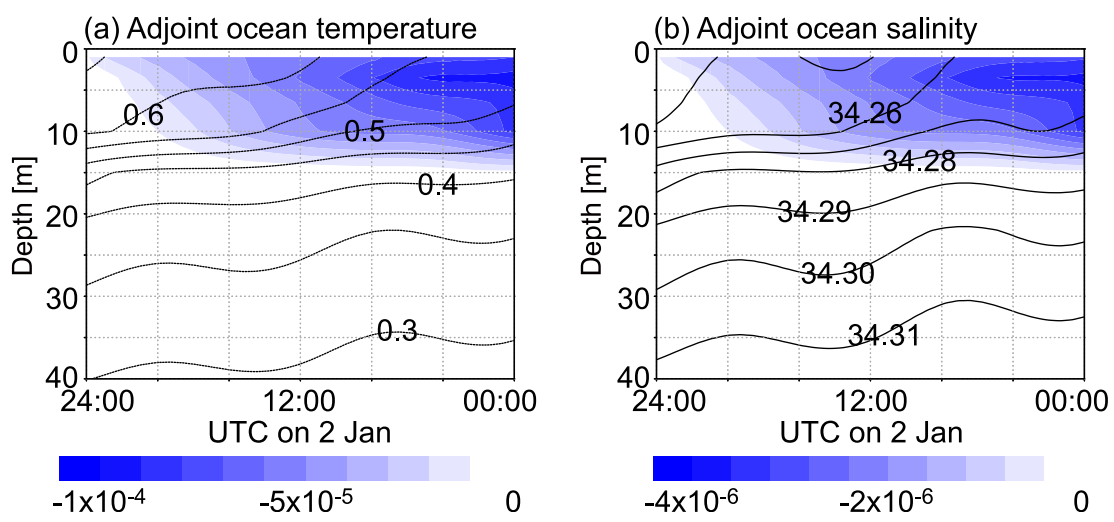

FIG. 5. Backward time evolutions of adjoint variables at point D in EXP-Dh: (a) $\tilde{T}$ and (b) $\tilde{S}$. Color shades in (a) and (b) indicate potential temperature and salinity, respectively, in the forward model.

heat for melting is smaller. In addition, some feedback occurs from these adjoint ocean variables to the adjoint sea ice variables, because, in the forward direction, the total amount of air-ocean fluxes depends on the openwater fraction (and, thus, the total sea ice area fraction) and also because the change in the ocean salinity due to the formation of ice from snow (as described above for categories 1 and 2) depends on the area fractions of ice of each category. Thus, $\tilde{T}$ and $\tilde{S}$ can affect $\widetilde{a_{n}}$ in the backward direction.

The dynamical effect can be investigated by conducting experiments that are the same as EXP-Da and EXP-Dh ("rheology inclusion" experiments, for simplicity), except with reduced dynamics, by eliminating the adjoints of the rheology and ridging schemes (EXP-DaX and EXP-DhX, respectively; "free drift" experiments). The results of these experiments were almost the same as those of EXP-Da and EXP-Dh. For example, the root-mean-square differences (RMSDs) in the fraction-weighted averages of the adjoint sea ice area fraction and thickness, defined in this study as $\tilde{a}=\sum_{n}\left(a_{n} \widetilde{a_{n}}\right) / \sum_{n} a_{n}$ and $\tilde{h}=\sum_{n}\left(a_{n} \widetilde{h_{n}}\right) / \sum_{n} a_{n}$, respectively, were $3.90 \times 10^{-5}$ and $1.98 \times 10^{-6}\left(6.67 \times 10^{-6}\right.$ and $\left.3.53 \times 10^{-6}\right)$, respectively, between EXP-Da and EXPDaX (EXP-Dh and EXP-DhX). These RMSDs are much smaller than the $\tilde{a}$ and $\tilde{h}$ values in EXP-Da and EXP-Dh (gray lines in Figs. 3 and 4). This reflects that the sea ice is mostly freely drifted without the effects of the internal stresses as expected. The rheology inclusion effects are highlighted in the following sections.

\section{b. Sensitivity in a region where full sea ice dynamics is important}

In the second set of experiments, initial values for adjoint sea ice variables were given at a point in the central Arctic Ocean $\left(80^{\circ} \mathrm{N}, 120^{\circ} \mathrm{W}\right.$; point $\mathrm{C}$ hereafter $)$, where the effects of the internal stresses are relatively strong.

At point $\mathrm{C}$, the total sea ice area fraction was 0.998 (Fig. 6a) and the grid-mean sea ice thickness was $4.50 \mathrm{~m}$ (Fig. 6c). Hence, the ocean was almost completely covered by sea ice. Relatively thick sea ice (categories 4 and 5) occupied an area fraction of 0.847 (Figs. 6b and $6 \mathrm{~d})$, and it can be inferred that sea ice in categories 1-3 had become thicker through ridging. At point $\mathrm{C}$, sea ice was moving southward at a speed of approximately $0.124 \mathrm{~m} \mathrm{~s}^{-1}$ (Fig. 6c). Changes in category 5 ice can be partly attributed to advection, because category 5 sea ice had smaller area fraction and larger thickness values in the north (upstream). Surface air temperature was about $-20^{\circ} \mathrm{C}$ (Fig. 6a). Accordingly, new sea ice was being created in the open-water area and added to category 1 ice (Figs. $6 \mathrm{~b}$ and $6 \mathrm{~d}$ ), maintaining a high total sea ice fraction despite the ridging effect. Hence, the freezing condition was another difference to point $\mathrm{D}$ in addition to the difference in the dynamical effects.

In EXP-Ca, an initial value of the adjoint sea ice area fraction of +0.01 was set at point C. As in EXP-Da (Fig. 3a), $\widetilde{a_{n}}$ values decreased during the backward integration (Fig. 7a), but the rates of decrease were greater in EXP-Ca than in EXP-Da; as a result $\tilde{a}$ was reduced to approximately $1 / 10$ of the initial value after backward integration for 1 day. Under the freezing condition, an increase of the (forward) area fraction reduces the surface cooling, which in turn decreases the new sea ice creation. Thus, the initial perturbation dissipates rather faster because the full ice cover would be achieved anyway. In the backward integration, this thermodynamical effect works to reduce the initialized value of the adjoint area fraction, leading to the greater rates of the $\tilde{a}$ decrease in EXP-Ca than those in EXP-D1. 
(a) Total SI fraction \& air temp
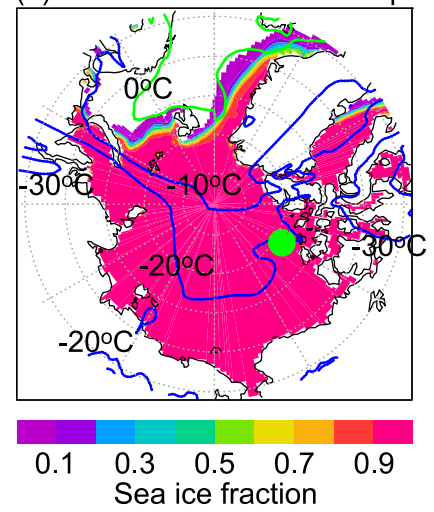

(c) SI thickness \& velocity

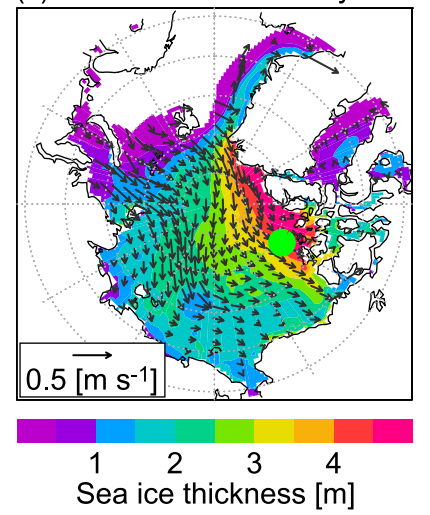

(b) SI fraction \& its change during 2 Jan

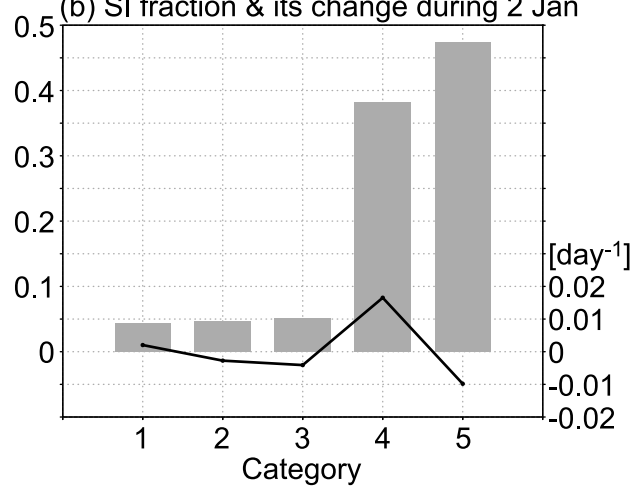

(d) SI thickness \& its change during 2 Jan

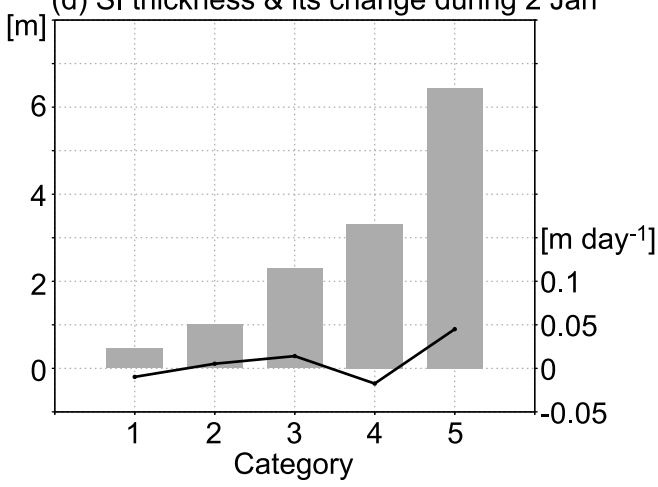

FIG. 6. As in Fig. 2, but for point C $\left[80^{\circ} \mathrm{N}, 120^{\circ} \mathrm{W}\right.$; green closed circle in (a) and (c)].

Another contribution from the internal stresses is described later. In addition, the initialized $\widetilde{a_{n}}$ values effectively generated negative $\widetilde{h_{n}}$ values, which had much larger magnitudes in the categories 4 and 5 than in categories 1-3 (Fig. 7b). This is because the decreases of thickness in the former categories effectively lead to the increases of area fraction in the latter categories. Note that $\widetilde{a_{n}}$ values are larger for smaller categories
(Fig. 7a). Unlike at point D (EXP-Da), no bottom melting effect was seen at point $\mathrm{C}$.

The next experiment (EXP-Ch) was the same as EXP$\mathrm{Ca}$, but an initial value of the adjoint sea ice thickness of +0.01 was set. In the backward integration, $\widetilde{h_{n}}$ values decreased from the initial values, as in EXP-Dh (except in category 4$)$, but the rates of decrease were greater, particularly in categories 4 and 5 (Fig. 8a). Positive $\widetilde{a_{n}}$ (a) Adjoint SI area fraction

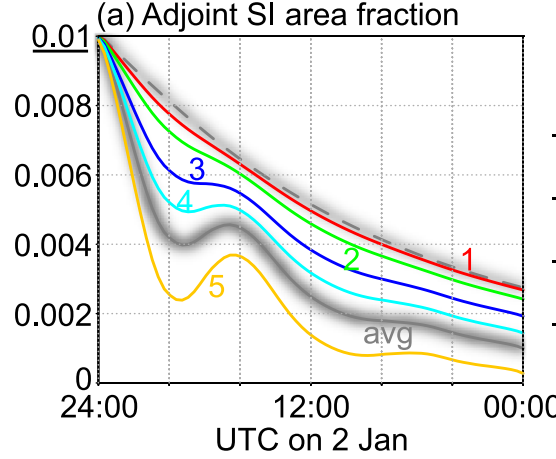

(b) Adjoint SI thickness

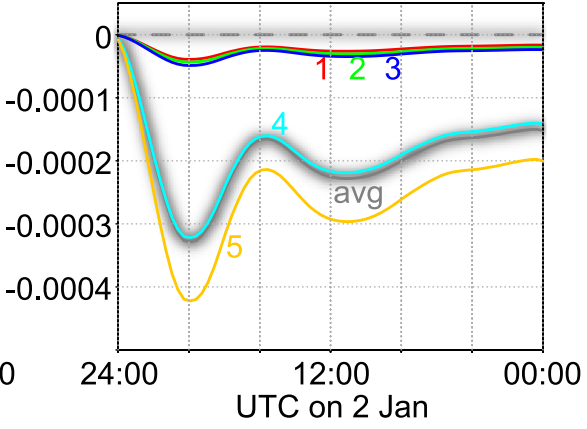

FIG. 7. Backward time evolutions of adjoint sea ice variables at point C in EXP-Ca: (a) $\widetilde{a_{n}}$ and (b) $\widetilde{h_{n}}$. Numbers by each line indicate categories; $\tilde{a}$ and $\tilde{h}$ values are also plotted for EXP-Ca (solid gray lines) and EXP-CaX (dashed gray lines). 
(a) Adjoint SI thickness

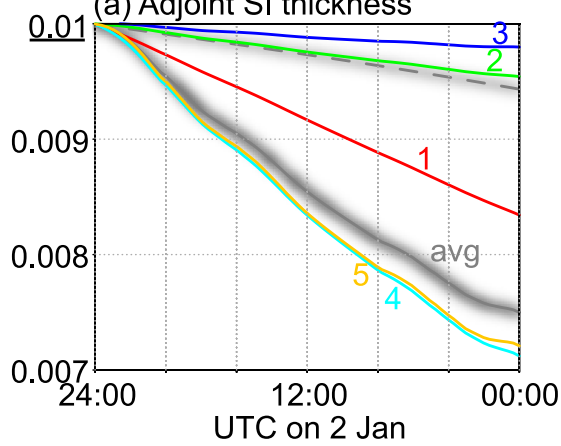

(b) Adjoint SI area fraction

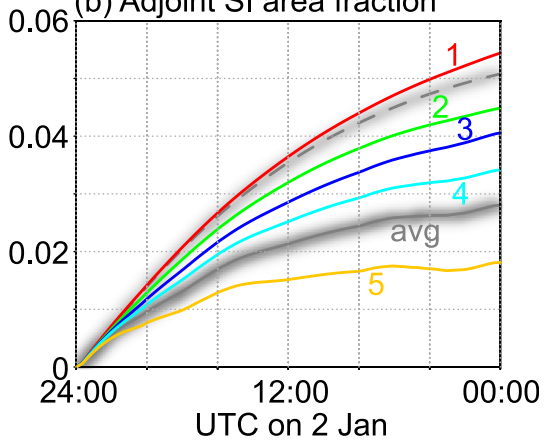

FIG. 8. Backward time evolutions of adjoint sea ice variables at point C in EXP-Ch: (a) $\widetilde{h_{n}}$ and (b) $\widetilde{a_{n}}$. Numbers by each line indicate categories; $\tilde{h}$ and $\tilde{a}$ values are also plotted for EXP-Ch (solid gray lines) and EXP-ChX (dashed gray lines).

values were forced by the initialized $\widetilde{h_{n}}$ value (Fig. 8 b), which became greater than the initialized value in EXP-Ca $(+0.01)$ within a few hours. This partly contributed to the $\widetilde{h_{n}}$ decreases since positive $\widetilde{a_{n}}$ works to reduce $\widetilde{h_{n}}$ as in EXP-Ca (Fig. 7).

To examine the influence of the reduced adjoint dynamics, we also conducted EXP-CaX and EXPChX, which were the same as EXP-Ca and EXP-Ch, respectively, but with reduced adjoint sea ice dynamics (free drift only). The rates of $\widetilde{a_{n}}$ decreases in EXP-CaX were smaller than those in EXP-Ca (Fig. 7a, dashed and solid gray lines), which resulted in $\widetilde{a_{n}}$ values about 3 times larger in EXP-CaX after backward integration for 1 day. The changes in $\widetilde{h_{n}}$ seen in EXP-Ca (Fig. 7b, solid gray line) almost disappeared without the adjoint rheology (EXP-CaX; dashed gray line; $\tilde{h}=-2.25 \times 10^{-9}$ at 0000 UTC). Thus, $\widetilde{a_{n}}$ generated $\widetilde{h_{n}}$ (with the opposite sign to $\widetilde{a_{n}}$ ) only when the adjoint rheology was included. In EXP-ChX, the tendency of the initialized $\tilde{h}$ value to decrease was weakened relative to that in EXP-Ch (Fig. 8a). Thus, the reduction rates of the initialized $\widetilde{a_{n}}$ or $\widetilde{h_{n}}$ were smaller without the adjoint rheology. On the other hand, the forced $\tilde{a}$ value was approximately doubled without the adjoint rheology (Fig. 8b).

The forward and adjoint sea ice velocity fields are shown in Fig. 9. For our model, we adopted an Arakawa B-grid coordinate system, so both ocean and sea ice velocities are defined at the corners of the grid cells where the sea ice area fraction and thickness, as well as the ocean potential temperature and salinity, are defined (e.g., solid pink rectangles in Fig. 9). The adjoint sea ice velocities in the free drift experiments showed convergent features (Figs. 9d and 9e). This result is intuitive because a positive change in the sea ice area fraction or thickness can be achieved by a convergent velocity field through advection (free drift). The greater adjoint sea ice velocities in EXP-ChX compared with EXP-CaX can be attributed to the greater $\widetilde{a_{n}}$ and $\widetilde{h_{n}}$ values in EXP-ChX (Figs. 7 and 8).

The adjoint sea ice velocities in EXP-Ca and EXPCh (i.e., with adjoint rheology; Figs. $9 \mathrm{~b}$ and $9 \mathrm{c}$ ) had much smaller magnitudes than those in EXP-CaX and (a) Forward SI velocity

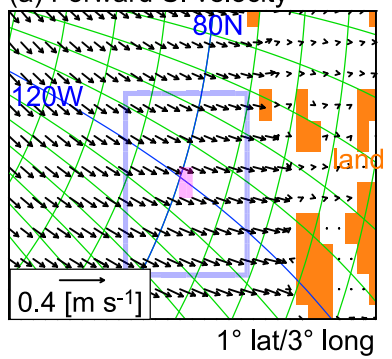

(b) EXP-Ca

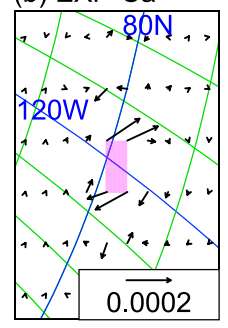

(c) EXP-Ch

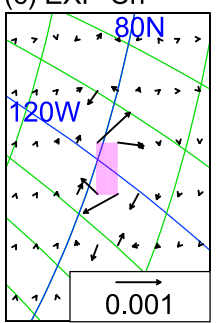

(d) EXP-CaX

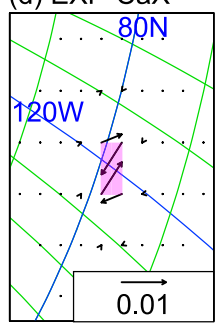

(e) EXP-ChX

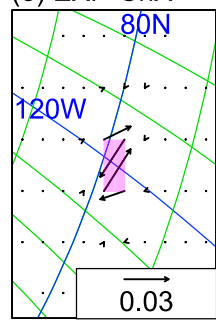

Adjoint SI velocity (vector scales are different)

FIG. 9. Distributions of forward and adjoint sea ice velocities averaged over the experimental period (1 day). (a) Forward sea ice velocities (common to all experiments). (b)-(e) Adjoint sea ice velocities in (b) EXP-Ca, (c) EXP-Ch, (d) EXP-CaX, and (e) EXP-ChX. Latitudinal and longitudinal lines for every $3^{\circ}$ zonally (positive upward) and every $1^{\circ}$ meridionally (positive leftward) are green, except $80^{\circ} \mathrm{N}$ and $120^{\circ} \mathrm{W}$, which are blue. Purple box in (a) indicates the region in which the adjoint sea ice velocities are shown in (b)-(e). The pink rectangle in each panel shows the grid box where the initial sensitivity was given. 

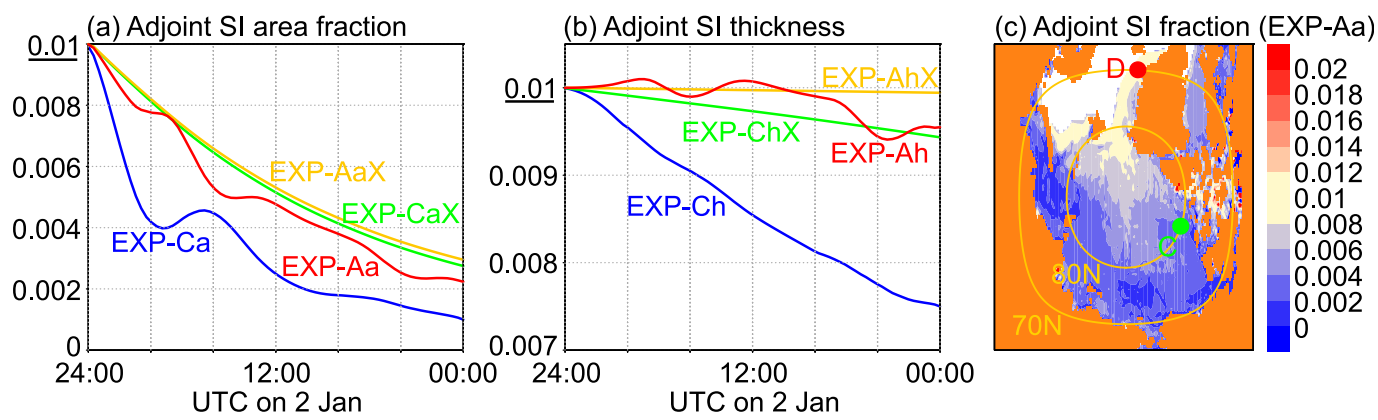

FIG. 10. (a) Backward time evolutions of $\tilde{a}$ at point $\mathrm{C}$ in the experiments with $\widetilde{a_{n}}$ initialized. (b) Backward time evolutions of $\tilde{h}$ in the experiments with $\widetilde{h_{n}}$ initialized. (c) Distribution of $\tilde{a}$ averaged over the experimental period in EXP-Aa. Points $\mathrm{D}\left(70^{\circ} \mathrm{N}, 18^{\circ} \mathrm{W}\right)$ and $\mathrm{C}\left(80^{\circ} \mathrm{N}, 120^{\circ} \mathrm{W}\right)$ are indicated by red and green circles, respectively.

EXP-ChX, respectively. In addition, divergent features can be seen, unlike in the free drift experiments. Although these features possibly contributed to the greater rates of reduction of the initialized $\widetilde{a_{n}}$ or $\widetilde{h_{n}}$ as described above, it was suggested that the adjoint sea ice velocities resulting from the initialized adjoint sea ice area fraction or thickness at a point (Fig. 9b) can be at least partly cancelled by the effect of neighboring values when initial values are given for an area instead of a single point. This effect is investigated in section $3 \mathrm{c}$.

\section{c. Horizontal interaction of sensitivities over the Arctic Ocean}

Interaction of sensitivities between the neighboring grids, particularly for the sea ice velocity, was suggested in the previous section. In this section, we investigate the effects of the horizontal interaction on the sensitivity evolution by using distributed initial values. The result also provides verification that the general features of the marginal ice zones and the central Arctic Ocean are captured at the points $\mathrm{D}$ and $\mathrm{C}$, respectively, as discussed in the previous sections. Thus, we examine the results of experiments in which initial values of +0.01 were given for the adjoint sea ice area fraction and thickness (EXP-Aa and EXP-Ah, respectively) for the whole Arctic Ocean, and for similar experiments, EXPAaX and EXP-AhX, respectively, with reduced adjoint sea ice dynamics (free drift only).

At point $\mathrm{D}\left(70^{\circ} \mathrm{N}, 18^{\circ} \mathrm{W}\right)$, where the effects of the internal stresses are small, although the time evolutions of $\widetilde{a_{n}}$ and $\widetilde{h_{n}}$ in EXP-Aa (not shown) were similar to those in EXP-Da, the decrease of $\widetilde{a_{n}}$ from the initialized value was smaller in EXP-Aa than in EXP-Da. The smaller decrease can be attributed to smaller advection and diffusion effects in EXP-Aa under the influence of neighboring values. Accordingly, the fraction-weighted averages $\tilde{a}$ and $\tilde{h}$ in EXP-Aa (for instance, 0.00985 and $8.76 \times 10^{-5}$, respectively, at 0000 UTC) were larger than those in EXP-Da $\left(0.00889\right.$ and $7.71 \times 10^{-5}$, respectively;
Fig. 3). However, the differences in the time evolutions of $\widetilde{a_{n}}$ and $\widetilde{h_{n}}$ at point $\mathrm{D}$ were trivial between EXP-Aa and EXP-AaX and between EXP-Ah and EXP-AhX (not shown) because the effects of the internal stresses are small at this point.

At point $\mathrm{C}\left(80^{\circ} \mathrm{N}, 120^{\circ} \mathrm{W}\right)$, where the effects of the internal stresses are relatively large, $\tilde{a}(\tilde{h})$ in EXP-Aa (EXP-Ah) decreased from the initialized value, as in EXP-Ca (EXP-Ch), but at a smaller rate (Figs. 10a and 10b). The smaller rate of decrease can be attributed partly to the influence of the values given in the surrounding grid cells, as discussed above for point $\mathrm{D}$. This effect can be seen in the differences between the free drift experiments (e.g., between EXP-CaX and EXPAaX; green and yellow lines). In addition, as discussed in section $3 b$, the adjoint sea ice velocities forced by the initialized $\widetilde{a_{n}}$ and $\widetilde{h}_{n}$ around point $\mathrm{C}$ differed greatly when the initial values were given for the whole region. Thus, the differences between the rheology inclusion experiments (e.g., between EXP-Ca and EXP-Aa; blue and red lines) reflect the effect of both 1) smaller decreases of initialized $\widetilde{a_{n}}$ and $\widetilde{h_{n}}$ and 2) large differences in the adjoint sea ice velocities, with the second effect being larger at point $\mathrm{C}$ than at point $\mathrm{D}$.

The decreases of the initialized $\tilde{a}$ (e.g., in EXP-Aa) were much greater than the decreases of the initialized $\tilde{h}$ (e.g., in EXP-Ah) (Fig. 10), although this feature is more noticeable at point $\mathrm{C}$ than at point $\mathrm{D}$, as discussed above. The horizontal distribution of $\tilde{a}$ values averaged over the experimental period (Fig. 10c) shows that the sensitivity information greatly dissipated during the day in a large part of the Arctic Ocean, except in marginal ice zones of the Atlantic section. On the same time scale, initialized $\tilde{h}$ values mostly remained or changes were relatively small (not shown). This result supports previous findings (e.g., Toyoda et al. 2011; BlanchardWrigglesworth et al. 2011) that sea ice thickness information is particularly important for correcting sea ice variability on this and longer time scales. On the 


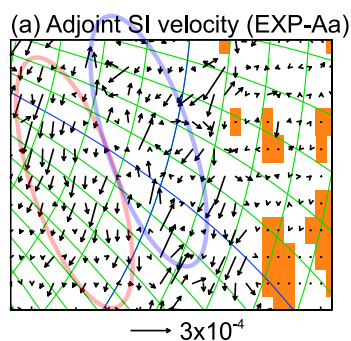

(d) Forward SI velocity

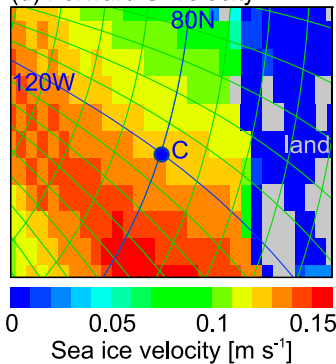

(g) Forward SI pressure

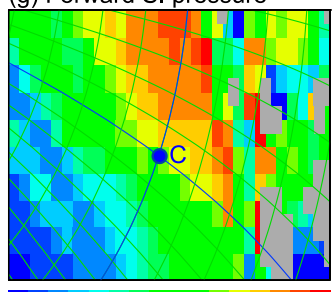

0.70 .80 .9111 .11 .21 .31 .4

Sea ice pressure $\left[10^{5} \mathrm{~N}\right]$

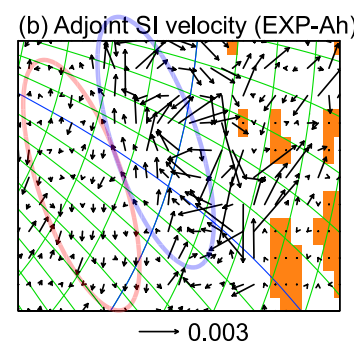

(e) Forward SI area fraction

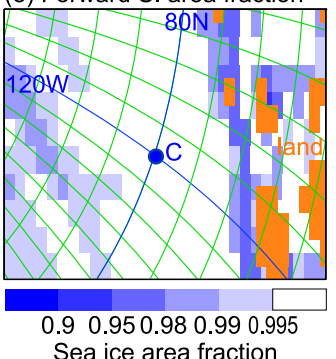

Sea ice area fraction

(h) Net area reduction rate

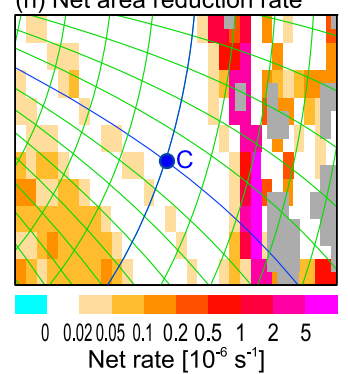

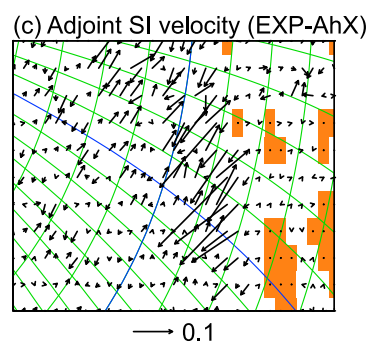

(f) Forward SI thickness

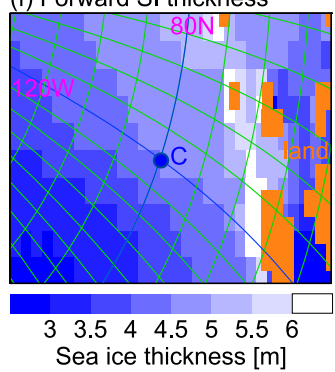

$\begin{array}{lllll}3.5 & 4 & 4.5 & 5 & 5.5 \\ \text { Sea ice thickness } & {[\mathrm{m}]}\end{array}$

FIG. 11. Distributions of adjoint sea ice velocities in (a) EXP-Aa, (b) EXP-Ah, and (c) EXP-AhX, normalized by using the magnitudes of the forward sea ice velocities (see text). Vector scales are indicated below the figures. Aligned structures were indicated by ellipses. Distributions of (d) forward sea ice velocity, (e) sea ice area fraction, (f) sea ice thickness, (g) sea ice pressure, and (h) net sea ice area reduction rate $\left(R_{\text {net }}\right)$ by ridging. Values are averaged over the experimental period. Latitudinal and longitudinal lines for every $3^{\circ}$ zonally (positive upward) and every $1^{\circ}$ meridionally (positive leftward) are green, except $80^{\circ} \mathrm{N}$ and $120^{\circ} \mathrm{W}$, which are blue. Note that the color scale intervals vary in (e) and (h).

other hand, the dissipative feature of the adjoint area fraction corresponds to the fact that sea ice concentration is relatively stable (close to 1 ) in the central Arctic Ocean because of freezing conditions.

We next investigated detailed structures of adjoint sea ice velocity and other dynamical variables in the same experiments, focusing on the region north of the Canadian Archipelago (which includes point $C$ ). Figures 11a-c show the normalized adjoint sea ice velocity fields, that is, the products of the adjoint velocities and the local magnitudes of the forward velocities (Fig. 11d) divided by a typical value over this region $(0.1 \mathrm{~m})$. This normalization was adopted with consideration of the relative importance of the sensitivities in relation to variabilities in the forward variables; even if the magnitude of the adjoint velocity is large, the sensitivity may be less important near land, where the forward velocity is less variable. Note that this will be reflected in the background errors in future state estimation experiment. As expected, the adjoint velocities forced at point $\mathrm{C}$ (Figs. 9b and 9c) were greatly altered by the effect of neighboring values, and, as a result, aligned adjoint velocity structures were visible in EXP-Aa and EXP-Ah. For example, adjoint velocities are westward and southwestward within the red ellipses (left side of Figs. 11a and 11b), whereas they are eastward and northeastward in the central area (blue ellipses in Figs. 11a and 11b).

In the free drift experiments such as EXP-AhX (Fig. 11c), however, the directions of the adjoint velocities differed from those described above and their magnitudes were much larger. This can be attributed to absence of the adjoint rheology. Thus, the internal stresses considered in the rheology suppressed the magnitudes of the adjoint sea ice velocity, as it suppresses the magnitudes of the sea ice velocity in the forward model. On the other hand, the adjoint sea ice velocities can be exaggerated without the feedback from the adjoint pressure field. This result indicates that for determining sensitivities with respect to 


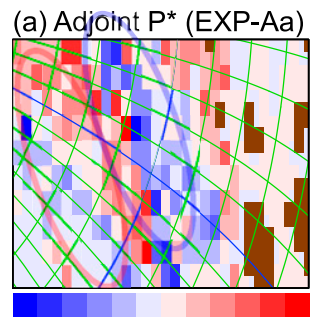

$\begin{array}{llllll}-8 & -4 & 0 & 4 & 8 & {\left[10^{-8}\right]}\end{array}$

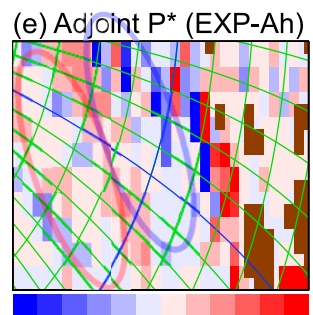

$\begin{array}{llll}-1.6-0.8 & 0 & 0.8 & 1.6\left[10^{-6}\right]\end{array}$

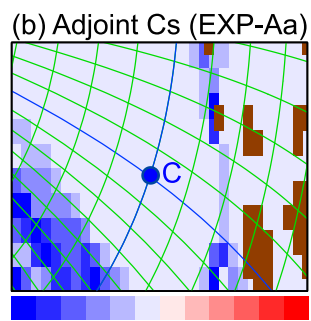

$\begin{array}{lllll}-4 & -2 & 0 & 2 & 4\left[10^{-8}\right]\end{array}$

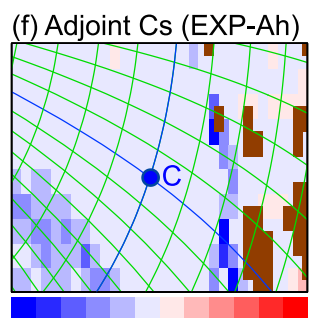

$\begin{array}{lllll}-4 & -2 & 0 & 2 & 4\left[10^{-7}\right]\end{array}$

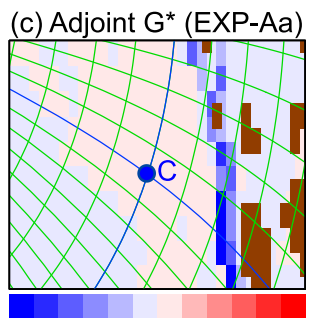

$\begin{array}{lllll}-8 & -4 & 0 & 4 & 8 \\ {\left[10^{-4}\right]}\end{array}$

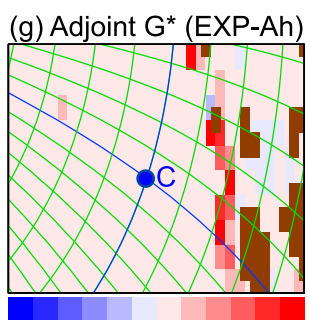

$\begin{array}{lllll}-4 & -2 & 0 & 2 & 4\left[10^{-3}\right]\end{array}$ (d) Adjoint Cd (EXP-Aa)

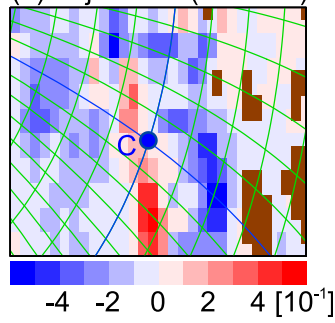

(h) Adjoint Cd (EXP-Ah)

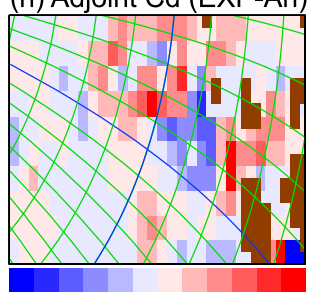

$\begin{array}{lllll}-8 & -4 & 0 & 4 & 8 \\ {\left[10^{1}\right]}\end{array}$

FIG. 12. Distributions of the adjoint nonprognostic variables (a),(e) $\widetilde{P^{*}},(\mathrm{~b}),(\mathrm{f}) \widetilde{C_{S}},(\mathrm{c}),(\mathrm{g}) \widetilde{G^{*}}$, and (d),(h) $\widetilde{C_{d}}$ for (a)-(d) EXP-Aa and (e)-(h) EXP-Ah. Values are accumulated over the experimental period and normalized by using the magnitudes of the forward sea ice velocities (Fig. 11d) as for Figs. 11a-c. Note that $P^{*}$ is the sea ice strength parameter, $C_{S}$ is the fraction of shear dissipation energy that contributes to ridge building, $G^{*}$ is the parameter representing the high end of the area fraction subject to ridging, and $C_{d}$ is the air-ice drag coefficient as defined in section $2 \mathrm{a}$.

sea ice velocity, it is important to consider the full sea ice dynamics including rheology.

Although the region around point $\mathrm{C}$ was almost fully covered by thick sea ice, as described in section $3 \mathrm{~b}$, horizontal differences can be seen in the distributions of the forward sea ice area fraction and thickness (Figs. 11e and 11f). In the nearshore region north of the Canadian Archipelago, sea ice was relatively thick (e.g., $>5 \mathrm{~m}$ ) and the sea ice area fraction was relatively low. The sea ice pressure field, calculated according to (5), shows that although pressure increased southeastward as the thickness increased (Fig. 11g), it decreased greatly in the nearshore region, reflecting the low area fraction. The distribution of the net rate of area loss $R_{\text {net }}$ as defined by (9) (Fig. 11h) indicates that ridging was enhanced in the nearshore region. Thus, the low sea ice area fraction and great sea ice thickness there can be attributed to ridging; ridging was also active in the northwestern region (Fig. 11h), but it was weaker than in the nearshore region. The regions with ridging in the northwestern region corresponded by and large to those with a relatively low sea ice area fraction (Fig. 11e).

Among distributions of the adjoint nonprognostic variables, those of $\widetilde{P^{*}}$ in EXP-Aa and EXP-Ah (Figs. 12a and 12e) exhibited a general pattern of positive values toward the north and west (within the red ellipses in Figs. 11a and 11b); negative values in the central region (blue ellipses); and positive values in the nearshore region. Because the forward sea ice velocities were mostly southward (Fig. 9a), these sensitivities with respect to $P^{*}$ are consistent with the southward components of the adjoint sea ice velocities in the north (within the red ellipse) enhancing sea ice pressure there, and their northward components in the central area (within the blue ellipse) reducing the sea ice pressure. The distribution patterns of $\tilde{C}$ were similar to those of $\widetilde{P^{*}}$ except with opposite sign [as in (12) and (13)], although the magnitudes differed (not shown).

Because the sea ice area fraction is reduced by ridging, negative sensitivities to the nonprognostic variables for the ridging process $\widetilde{C_{S}}$ and $\widetilde{G^{*}}$ (adjoint variables for the fraction of shear dissipation energy that contributes to ridge building $C_{S}$ and the high end of the area fraction subject to ridging $G^{*}$ ) were seen in EXP-Aa (Figs. 12b and $12 \mathrm{c}$ ), particularly in regions where the forward sea ice area fraction was relatively low. In EXP-Ah, the $\widetilde{C_{S}}$ distribution pattern (Fig. 12f) was similar to the pattern in EXP-Aa, but, different from EXP-Aa, some positive $G^{*}$ values were seen, especially in the nearshore region (Fig. 12g). The positive values reflect the fact that the weighting function $b(h)(7)$ is applied to larger $h$ when $G^{*}$ is larger, leading to the production of thicker sea ice by ridging.

The distribution patterns of both adjoint wind stress and adjoint surface wind speed (not shown) were similar in those of the adjoint sea ice velocity shown in Figs. 11a and $11 \mathrm{~b}$, but those of the adjoint air-ice drag coefficients were different (Figs. 12d and 12h). The distributions 
of these adjoint variables were possibly influenced by synoptic-scale atmospheric variability in the forward surface forcing field.

In this study, the sensitivities with respect to the nonprognostic variables were accumulated over the experimental period (1 day). In a future long-term experiment, although several issues should be addressed for conducting it, synoptic-scale variability (Figs. 12d and $12 \mathrm{~h}$ ) could be suppressed by setting an even longer estimation period (e.g., 1 month).

\section{Discussion}

Although the adjoint sea ice models did not consider the adjoint of the rheology in several previous studies, this exclusion does not decrease their value, because exact linear models are known to sometimes generate undesirably strong sensitivities, as discussed by Sugiura et al. (2014). Accordingly, in the adjoint of OGCMs, nonlinear parameterizations such as vertical mixing schemes have been excluded (e.g., Jiang et al. 2002; Weaver et al. 2003; Sugiura et al. 2008; Liu et al. 2012). In other approaches, the model resolution has been reduced or diffusivities have been increased artificially, in the adjoint model, to suppress the exponentially growing sensitivities (e.g., Köhl and Willebrand 2002; Hoteit et al. 2005). Because sea ice rheology models are highly nonlinear, with internal stresses and viscous parameters predicted as well as velocities in the rheology, much smaller time steps have been adopted than in the other parts of the OGCMs (e.g., Hunke and Dukowicz 1997). The exclusion of the adjoint of the rheology was, therefore, a reasonable compromise. In this study, we found a method to avoid the generation of too strong sensitivities while maintaining the adjoint of the main stress equations of the elastic-viscous-plastic rheology model that might be applicable to the viscousplastic rheology model (Hibler 1979) used in previous adjoint sea ice models. Although Kauker et al. (2009) obtained reasonable sensitivities of summer sea ice thickness with respect to wind stresses, Koldunov et al. (2017) recently reported that it is difficult to associate surface wind corrections obtained from sensitivity experiments with particular large-scale sea ice changes. Use of the adjoint sea ice rheology might improve consistency of the sensitivities.

The time window of the sensitivity experiments, which was only 1 day in this study, needs to be extended for a state estimation experiment on a seasonal or longer time scale. In addition, the data-model misfits would distribute complicatedly in such a realistic case. However, the relatively low computational cost as well as the simple settings of the initial conditions enabled us to carry out several experiments for investigating the detailed differences between the experiments with physical interpretations to evaluate the effectiveness of our method. Hence, we consider this study to be an important step toward a 4DVAR experiment conducted for a longer period, which requires multiple iterations of the forward and adjoint integration cycle and thus has a high computational cost. Note that issues other than the computational cost would arise when the period during the specific day in winter is extended. Detection and resolution of these potential issues remain for future work.

An improved ocean-sea ice state estimation from existing observations and state-of-the-art dynamical models would be possible through a 4DVAR data assimilation approach with the sea ice adjoint model including the rheology. Previous studies (e.g., Miller et al. 2006) have used sea ice concentration and velocity data based on satellite observations. The results of this study suggest that assimilation of such data by a 4DVAR method requires full adjoint sea ice dynamics including the rheology.

In addition, retrievals of sea ice thickness with enhanced reliability from satellite observations have recently become available (e.g., Laxon et al. 2013). The importance of sea ice thickness information has been recognized (e.g., Lindsay et al. 2012), and our result suggests that the initialized adjoint sea ice thickness has a larger effect than the initialized sea ice area fraction in several regions. Furthermore, recent studies have reported relatively large negative biases of sea ice thickness in several ocean-sea ice reanalyses (including ours) in the Canadian basin, in particular near the Canadian Archipelago (e.g., Chevallier et al. 2017; Uotila et al. 2019). Although this study focused on the Arctic Ocean, those previous studies also indicated the necessity of enhanced reproduction of the ocean-sea ice fields in both hemispheres. Assimilation of satellite-based sea ice concentration, velocity, and thickness data by a 4DVAR method would improve the global ocean-sea ice reanalyses.

\section{Summary}

We developed an adjoint model for the sea ice part of our institute's OGCM (MRI.COM; Tsujino et al. 2017). The adjoint sea ice dynamics includes rheology (Hunke and Dukowicz 1997), which had previously been excluded from adjoint models to avoid instability. We eventually merged our model with the main adjoint model developed previously (Usui et al. 2015) to construct a global ocean-sea ice adjoint model. We conducted a series of sensitivity experiments with initial 
(nonzero) values given for the adjoint sea ice area fraction and thickness to investigate the behavior of the adjoint model, in particular, the effects of improvements of the sea ice dynamics in the new adjoint sea ice model.

In a marginal ice zone, where the effects of the internal stresses are relatively small, the initialized adjoint sea ice area fraction was generally persistent, although it gradually decreased due to advection and diffusion processes. It generated the adjoint sea ice thickness mainly in the thinnest category, where the bottom melting process reduced the sea ice area fraction in the forward integration. The initialized adjoint thickness was also generally persistent and generated the adjoint potential temperature and salinity in the ocean surface layer whose sign was opposite to the initialized value. These features were common to the experiments with and without the adjoint rheology, consistent with the previous view (e.g., Haas 2003) that the sea ice is mostly freely drifted in the marginal ice zones.

In the central Arctic Ocean, where the effects of the internal stresses are important, the initialized adjoint sea ice area fraction generated a time change in the adjoint sea ice thickness whose sign was opposite to the initialized value, while the initialized adjoint sea ice thickness generated a time change in the adjoint sea ice area fraction whose sign was the same as the initialized value. The magnitudes of initialized adjoint area fraction or thickness were reduced over time and the rates of reduction were greater when the full adjoint sea ice dynamics was utilized. The latter was considered to reflect the effects of the rheology and ridging in determining the area fraction and thickness. In other words, these variables are more persistent under the free-drift approximation.

In addition, the adjoint sea ice velocities generated by the influence of the adjoint sea ice area fraction and thickness differed remarkably in both direction and magnitude between the experiments with full adjoint sea ice dynamics (including the rheology) and those with reduced adjoint sea ice dynamics (free drift only) (Fig. 11). The internal stresses considered in the rheology suppressed the magnitudes of the adjoint sea ice velocity, as it suppresses the magnitudes of the sea ice velocity in the forward model. Moreover, the sensitivities with respect to nonprognostic variables associated with the sea ice dynamics (e.g., $P^{*}$ and surface winds) could be deduced from the adjoint sea ice dynamics. The usefulness of these sensitivities should be demonstrated by evaluating the reproduction of future global oceansea ice state estimation through the 4DVAR method.

In constructing the adjoint sea ice model, we simplified the adjoint of the equations for viscosities in the rheology (by excluding dependency of the key variable $\tilde{\Delta}$ on the adjoint viscosities $\tilde{\zeta}$ and $\tilde{\eta}$ in the adjoint model). This simplification might deform the sensitivity structure. However, the adjoint sea ice stress and velocity fields can interact with the adjoint sea ice fraction and thickness fields through the rheology and ridging parameterization in the adjoint model, and the inclusion of the adjoint rheology had a large impact on the results of the adjoint model experiments, particularly in the adjoint sea ice velocity field with the ratio of magnitudes up to $O\left(10^{2}\right)$ (Fig. 11). These results suggested a potential for providing an improved global atmosphere-oceansea ice state estimation through a four-dimensional variational approach with the adjoint sea ice model as developed in this study.

Acknowledgments. We are indebted to D. Hotta (MRI) and T. Toyota (Low Temperature Institute, Hokkaido University) for helpful discussions. Constructive comments provided by anonymous reviewers improved the article. We acknowledge Y. Tsutsumi and M. Ishii, the previous and present Director-General of the Oceanography and Geochemistry Research Department of the MRI, for their encouragement, which helped us to complete this study. We also thank S. Duhon (ELSS Inc.) for her critical reading of our paper. This research was partly supported by the study on Arctic climate predictability (Theme 5) of the Arctic Challenge for Sustainability (ArCS) project funded by the Japanese Ministry of Education, Culture, Sports, Science and Technology and by the Japan Society for the Promotion of Science (KAKENHI Grant 16K17805).

\section{REFERENCES}

Awaji, T., S. Masuda, Y. Ishikawa, N. Sugiura, T. Toyoda, and T. Nakamura, 2003: State estimation of the North Pacific Ocean by a four-dimensional variational data assimilation experiment. J. Oceanogr., 59, 931-943, https://doi.org/10.1023/ B:JOCE.0000009582.24737.6f.

Blanchard-Wrigglesworth, E., C. M. Bitz, and M. M. Holland, 2011: Influence of initial conditions and climate forcing on predicting Arctic sea ice. Geophys. Res. Lett., 38, L18503, https:// doi.org/10.1029/2011GL048807.

Boyer, T. P., and Coauthors, 2013: World Ocean Database 2013. NOAA Atlas NESDIS 72, NOAA, 209 pp.

Chevallier, M., and Coauthors, 2017: Intercomparison of the Arctic sea ice cover in global ocean-sea ice reanalyses from the ORA-IP project. Climate Dyn., 49, 1107-1136, https://doi.org/ 10.1007/s00382-016-2985-y.

Colony, R., and A. S. Thorndike, 1980: The horizontal coherency of the motion of summer Arctic sea ice. J. Phys. Oceanogr., 10, 1281-1289, https://doi.org/10.1175/1520-0485(1980)010<1281: THCOTM $>2.0 . \mathrm{CO} ; 2$.

Fenty, I., and P. Heimbach, 2013: Coupled sea ice-ocean-state estimation in the Labrador Sea and Baffin Bay. J. Phys. Oceanogr., 43, 884-904, https://doi.org/10.1175/JPO-D-12-065.1. 
Flato, G. M., and W. D. Hibler III, 1995: Ridging and strength in modeling the thickness distribution of Arctic sea ice. J. Geophys. Res., 100, 18611-18 626, https://doi.org/10.1029/95JC02091.

Fujii, Y., and Coauthors, 2018: Ocean data assimilation systems in JMA and their representation of SST and sea ice fields. Workshop on observations and analysis of sea-surface temperature and sea ice for NWP and Climate Applications, Reading, United Kingdom, ECMWF, https://www.ecmwf.int/en/learning/ workshops/workshop-observations-and-analysis-sea-surfacetemperature-and-sea-ice-nwp-and-climate.

Giering, R., and T. Kaminski, 1998: Recipes for adjoint code construction. ACM Trans. Math. Software, 24, 437-474, https:// doi.org/10.1145/293686.293695.

Good, S. A., M. J. Martin, and N. A. Rayner, 2013: EN4: Quality controlled ocean temperature and salinity profiles and monthly objective analyses with uncertainty estimates. J. Geophys. Res. Oceans, 118, 6704-6716, https://doi.org/10.1002/2013JC009067.

Haas, C., 2003: Dynamics versus thermodynamics: The sea ice thickness distribution. Sea Ice, D. N. Thomas and G. S. Dieckmann, Eds., Blackwell Publishing Ltd., 113-151.

Hibler, W. D., III, 1979: A dynamic thermodynamic sea ice model. J. Phys. Oceanogr., 9, 815-846, https://doi.org/10.1175/15200485(1979)009<0815:ADTSIM>2.0.CO;2.

— , and J. E. Walsh, 1982: On modeling seasonal and interannual fluctuations of Arctic sea ice. J. Phys. Oceanogr., 12, 1514-1523, https://doi.org/10.1175/1520-0485(1982)012<1514:OMSAIF> 2.0.CO;2.

Hoteit, I., B. D. Cornuelle, A. Köhl, and D. Stammer, 2005: Treating strong adjoint sensitivities in tropical eddy-permitting variational data assimilation. Quart. J. Roy. Meteor. Soc., 131, 3659-3682, https://doi.org/10.1256/qj.05.97.

Hunke, E. C., and J. K. Dukowicz, 1997: An elastic-viscous-plastic model for sea ice dynamics. J. Phys. Oceanogr., 27, 1849-1867, https://doi.org/10.1175/1520-0485(1997)027<1849:AEVPMF> 2.0.CO;2.

— Model documentation and software user's manual version 4.1. Tech. Rep. LA-CC-06-012, Los Alamos National Laboratory, $76 \mathrm{pp}$.

Jiang, Z., M. Kamachi, and H. Wang, 2002: The improvement made by a modified TLM in 4DVAR with a geophysical boundary layer model. Adv. Atmos. Sci., 19, 563-582, https://doi.org/ 10.1007/s00376-002-0001-4.

Kalnay, E., Y. Ota, T. Miyoshi, and J. Liu, 2012: A simpler formulation of forecast sensitivity to observations: Application to ensemble Kalman filters. Tellus, 64A, 18462, https://doi.org/ 10.3402/tellusa.v64i0.18462.

Kauker, F., 2015: Report on the assessment of forecast skill. Rep. D1.83, Project 265863, Arctic Climate Change Economy and Society (ACCESS), 41 pp., http://www.access-eu.org/ modules/resources/download/access/Deliverables/D1-83OASys-final.pdf.

- - , T. Kaminski, M. Karcher, R. Giering, R. Gerdes, and M. Voßbeck, 2009: Adjoint analysis of the 2007 all time Arctic sea-ice minimum. Geophys. Res. Lett., 36, L03707, https://doi.org/10.1029/2008GL036323.

Kobayashi, S., and Coauthors, 2015: The JRA-55 reanalysis: General specifications and basic characteristics. J. Meteor. Soc. Japan, 93, 5-48, https://doi.org/10.2151/jmsj.2015-001.

Köhl, A., and J. Willebrand, 2002: An adjoint method for the assimilation of statistical characteristics into eddy-resolving ocean models. Tellus, 54A, 406-425, https://doi.org/10.1034/ j.1600-0870.2002.01294.x.
Koldunov, N. V., A. Köhl, N. Serra, and D. Stammer, 2017: Sea ice assimilation into a coupled ocean-sea ice model using its adjoint. Cryosphere, 11, 2265-2281, https://doi.org/10.5194/ tc-11-2265-2017.

Kurihara, Y., T. Sakurai, and T. Kuragano, 2006: Global daily sea surface temperature analysis using data from satellite microwave radiometer, satellite infrared radiometer and in-situ observations (in Japanese). Wea. Bull., 73, 1-18.

Large, W. G., and S. G. Yeager, 2009: The global climatology of an interannually varying air sea flux data set. Climate Dyn., 33, 341-364, https://doi.org/10.1007/s00382-008-0441-3.

Laxon, S. W., and Coauthors, 2013: CryoSat-2 estimates of Arctic sea ice thickness and volume. Geophys. Res. Lett., 40, 732-737, https://doi.org/10.1002/grl.50193.

Lindsay, R., and Coauthors, 2012: Seasonal forecasts of Arctic sea ice initialized with observations of ice thickness. Geophys. Res. Lett., 39, L21502, https://doi.org/ 10.1029/2012GL053576.

—- M. Wensnahan, A. Schweiger, and J. Zhang, 2014: Evaluation of seven different atmospheric reanalysis products in the Arctic. J. Climate, 27, 2588-2606, https://doi.org/10.1175/ JCLI-D-13-00014.1.

Lipscomb, W. H., 2001: Remapping the thickness distribution in sea ice models. J. Geophys. Res., 106, 13 989-14000, https:// doi.org/10.1029/2000JC000518.

Liu, C., A. Köhl, and D. Stammer, 2012: Adjoint-based estimation of eddy-induced tracer mixing parameters in the global ocean. J. Phys. Oceanogr., 42, 1186-1206, https://doi.org/ 10.1175/JPO-D-11-0162.1.

McPhee, M., 2008: Air-Ice-Ocean Interaction: Turbulent Ocean Boundary Layer Exchange Processes. Springer, 215 pp.

Mellor, G. L., and L. Kantha, 1989: An ice-ocean coupled model. J. Geophys. Res., 94, 10 937-10 954, https://doi.org/10.1029/ JC094iC08p10937.

Miller, P. A., S. W. Laxon, D. L. Feltham, and D. J. Cresswell, 2006: Optimization of a sea ice model using basinwide observations of Arctic sea ice thickness, extent, and velocity. J. Climate, 19, 1089-1108, https://doi.org/10.1175/JCLI3648.1.

Murray, R. J., 1996: Explicit generation of orthogonal grids for ocean models. J. Comput. Phys., 126, 251-273, https://doi.org/ 10.1006/jcph.1996.0136.

Nguyen, A., D. Menemenlis, and R. Kwok, 2011: Arctic ice-ocean simulation with optimized model parameters: Approach and assessment. J. Geophys. Res., 116, C04025, https://doi.org/ 10.1029/2010JC006573.

Pellerin, P., H. Ritchie, S. J. Saucier, F. Roy, S. Desjardins, M. Valin, and V. Lee, 2004: Impact of a two-way coupling between an atmospheric and an ocean-ice model over the Gulf of St. Lawrence. Mon. Wea. Rev., 132, 13791398, https://doi.org/10.1175/1520-0493(2004)132<1379: IOATCB $>2.0 . \mathrm{CO} ; 2$.

Serreze, M. C., and R. G. Barry, 2005: The Arctic Climate System. Cambridge University Press, 385 pp.

Steele, M., J. Zhang, D. Rothrock, and H. Stern, 1997: The force balance of sea ice in a numerical model of the Arctic Ocean. J. Geophys. Res., 102, 21 061-21 079, https://doi.org/10.1029/ 97JC01454.

Sugiura, N., T. Awaji, S. Masuda, T. Mochizuki, T. Toyoda, T. Miyama, H. Igarashi, and Y. Ishikawa, 2008: Development of a four-dimensional variational coupled data assimilation system for enhanced analysis and prediction of seasonal to interannual climate variations. J. Geophys. Res., 113, C10017, https://doi.org/10.1029/2008JC004741. 
, S. Masuda, Y. Fujii, M. Kamachi, Y. Ishikawa, and T. Awaji, 2014: A framework for interpreting regularized state estimation. Mon. Wea. Rev., 142, 386-400, https://doi.org/10.1175/MWRD-12-00231.1.

Thorndike, A. S., D. A. Rothrock, G. A. Maykut, and R. Colony, 1975: The thickness distribution of sea ice. J. Geophys. Res., 80, 4501-4513, https://doi.org/10.1029/JC080i033p04501.

Toyoda, T., and Coauthors, 2011: Impact of the assimilation of sea ice concentration data on an atmosphere-ocean-sea ice coupled simulation of the Arctic Ocean climate. SOLA, 7, 37-40, https://doi.org/10.2151/sola.2011-010.

—, Y. Fujii, T. Yasuda, N. Usui, T. Iwao, T. Kuragano, and M. Kamachi, 2013: Improved analysis of seasonal-interannual fields using a global ocean data assimilation system. Theor. Appl. Mech. Japan, 61, 31-48.

K. Ogawa, T. Kuragano, H. Tsujino, and M. Kamachi, 2016: Data assimilation of sea ice concentration into a global ocean-sea ice model with corrections for atmospheric forcing and ocean temperature fields. J. Oceanogr., 72, 235-262, https://doi.org/10.1007/s10872-015-0326-0.

Tsujino, H., M. Hirabara, H. Nakano, T. Yasuda, T. Motoi, and G. Yamanaka, 2011: Simulating present climate of the global ocean-ice system using the Meteorological Research Institute Community Ocean Model (MRI.COM): Simulation characteristics and variability in the Pacific sector. J. Oceanogr., 67, 449-479, https://doi.org/10.1007/s10872-011-0050-3.

, H. Nakano, K. Sakamoto, S. Urakawa, M. Hirabara, H. Ishizaki, and G. Yamanaka, 2017: Reference manual for the Meteorological Research Institute Community Ocean Model (MRI.COM) version 4. Tech. Rep. 80, Meteorological Research Institute, 284 pp., https://doi.org/10.11483/ mritechrepo.80.
— , and Coauthors, 2018: JRA-55 based surface dataset for driving ocean-sea-ice models (JRA55-do). Ocean Modell., 130, 79-139, https://doi.org/10.1016/j.ocemod.2018.07.002.

Uotila, P., and Coauthors, 2019: An assessment of ten ocean reanalyses in the polar regions. Climate Dyn., 52, 1613-1650, https://doi.org/10.1007/s00382-018-4242-z.

Urakawa, S. L., H. Tsujino, H. Nakano, K. Sakamoto, and G. Yamanaka, 2017: Global ocean model development for CMIP6 in Meteorological Research Institute and its performance in reproducing ocean general circulation. JpGU-AGU Joint Meeting 2017, Makuhari, Japan, Japan Geophys. Union, AOS19-03, https://confit.atlas.jp/guide/event/ jpguagu2017/subject/AOS19-03/tables.

Usui, N., S. Ishizaki, Y. Fujii, H. Tsujino, T. Yasuda, and M. Kamachi, 2006: Meteorological Research Institute multivariate ocean variational estimation (MOVE) system: Some early results. Adv. Space Res., 37, 806-822, https:// doi.org/10.1016/j.asr.2005.09.022.

_- Y. Fujii, K. Sakamoto, and M. Kamachi, 2015: Development of a four-dimensional variational assimilation system for coastal data assimilation around Japan. Mon. Wea. Rev., 143, 3874-3892, https://doi.org/10.1175/MWR-D-14-00326.1.

— , and Coauthors, 2017: Four-dimensional variational ocean reanalysis: A 30-year high-resolution dataset in the western North Pacific (FORA-WNP30). J. Oceanogr., 73, 205-233, https://doi.org/10.1007/s10872-016-0398-5.

Weaver, A., J. Vialard, and D. L. Anderson, 2003: Three- and four-dimensional variational assimilation with a general circulation model of the tropical Pacific Ocean. Part I: Formulation, internal diagnostics, and consistency check. Mon. Wea. Rev., 131, 1360-1378, https://doi.org/10.1175/ 1520-0493(2003)131<1360:TAFVAW>2.0.CO;2. 